\title{
Cadherin-11 blockade reduces inflammation-driven fibrotic remodeling and improves outcomes after myocardial infarction
}

\author{
Alison K. Schroer, ${ }^{1}$ Matthew R. Bersi, ${ }^{1}$ Cynthia R. Clark, ${ }^{1}$ Qinkun Zhang, ${ }^{2}$ Lehanna H. Sanders, ${ }^{2}$ \\ Antonis K. Hatzopoulos, ${ }^{2}$ Thomas L. Force, ${ }^{2}$ Susan M. Majka, ${ }^{3}$ Hind Lal, ${ }^{2}$ and W. David Merryman ${ }^{1}$ \\ 'Department of Biomedical Engineering, 'Department of Cardiovascular Medicine, and ${ }^{3}$ Department of Allergy, Pulmonary, \\ and Critical Care Medicine, Vanderbilt University, Nashville, Tennessee, USA.
}

\begin{abstract}
Over one million Americans experience myocardial infarction (MI) annually, and the resulting scar and subsequent cardiac fibrosis gives rise to heart failure. A specialized cell-cell adhesion protein, cadherin-11 (CDH11), contributes to inflammation and fibrosis in rheumatoid arthritis, pulmonary fibrosis, and aortic valve calcification but has not been studied in myocardium after MI. MI was induced by ligation of the left anterior descending artery in mice with either heterozygous or homozygous knockout of CDH11, wild-type mice receiving bone marrow transplants from Cdh11deficient animals, and wild-type mice treated with a functional blocking antibody against CDH11 (SYN0012). Flow cytometry revealed significant CDH11 expression in noncardiomyocyte cells after MI. Animals given SYN0012 had improved cardiac function, as measured by echocardiogram, reduced tissue remodeling, and altered transcription of inflammatory and proangiogenic genes. Targeting CDH11 reduced bone marrow-derived myeloid cells and increased proangiogenic cells in the heart 3 days after MI. Cardiac fibroblast and macrophage interactions increased IL- 6 secretion in vitro. Our findings suggest that CDH11-expressing cells contribute to inflammation-driven fibrotic remodeling after $\mathrm{MI}$ and that targeting $\mathrm{CDH} 11$ with a blocking antibody improves outcomes by altering recruitment of bone marrow-derived cells, limiting the macrophage-induced expression of IL-6 by fibroblasts and promoting vascularization.
\end{abstract}

Conflict of interest: The authors have declared that no conflict of interest exists.

Copyright: (c) 2019, American Society for Clinical Investigation.

Submitted: July 3, 2019

Accepted: August 21, 2019

Published: September १९, 2019.

Reference information: /CI Insight. 2019;4(18):e131545.

https://doi.org/10.1172/jici.

insight.131545.

\section{Introduction}

Every year over one million Americans experience a myocardial infarction (MI), which significantly reduces cardiac function and potentiates the progression to heart failure by increasing the risk of recurrent infarctions (1). The process of infarct healing requires complex interactions between resident and recruited cells, which must coordinate the clearance and replacement of damaged tissue with a stable and robust collagen scar to prevent cardiac rupture.

Immune cells - including neutrophils, monocytes, and macrophages, among others - are recruited from the blood within the first few hours after MI and critically participate in the healing and remodeling process. Resident cardiac macrophages also participate in stabilizing the heart after cardiac injury (2). Following the successful clearance of necrotic tissue and cell debris by immune cells, resident mesenchymal cells — including cardiac fibroblasts (CFs) - proliferate and differentiate into an activated, hypersecretory, hypercontractile, tissue remodeling phenotype known as myofibroblasts. This highly cellularized stage of cardiac remodeling, termed the granulation phase, is characterized by the resolution of inflammatory signaling and the transition to fibrotic remodeling and scar formation by activated myofibroblasts. In the following weeks, myofibroblasts deposit and remodel collagen into a compact scar, which can sustain the biomechanical integrity of the myocardial wall (3). However, excess inflammation and reparative activity can ultimately lead to expansion of the infarct area and further diminished cardiac function $(4,5)$.

Development of treatment strategies for MI is made particularly challenging by the precise and necessary timing of both chemical signals and cellular activity throughout these phases of remodeling. Many treatments targeting specific growth factor cascades have failed to maintain the delicate balance between 
necessary and excessive inflammation and fibrosis and often have adverse side effects on the surviving cardiomyocytes (CMs), causing additional loss of contractile potential (6-8).

Cadherin-11 (CDH11) is a cell-cell adhesion protein expressed by inflammatory cells and activated fibroblast-like cells in multiple inflammatory and fibrotic disease models — including rheumatoid arthritis, pulmonary fibrosis, and aortic valve calcification (9-11) - but its function in infarct healing has not yet been studied. CDH11 (or OB-cadherin) was originally described in osteoblasts and has been shown to affect cell migration and exfiltration in cancer studies $(12,13)$, but the role of CDH11 in bone marrow-derived cell (BMDC) recruitment and CF contractility in the heart has not been studied. CDH11 expression has been observed in CFs but is minimally expressed by CMs in culture and has not been well studied in the myocardium in vivo (14). CDH11 engagement promotes the expression of the proinflammatory cytokine IL-6 as well as profibrotic signaling factors and myofibroblast markers, such as TGF- $\beta 1$, in diseased joints, lungs, and heart valves $(9-11,15,16)$. Thus, we hypothesized that genetic and pharmacologic targeting of CDH11 after MI would reduce inflammation-driven fibrotic scar expansion and improve cardiac outcomes.

\section{Results}

To establish the therapeutic potential of targeting CDH11 in the heart after MI, we first wanted to identify the cardiac cell populations on which CDH11 is expressed. Flow cytometric analysis of non-CM cardiac cell populations - including cardiac endothelial cells (CECs: $\left.\mathrm{CD} 45^{-} \mathrm{CD} 31^{+}\right)$, cardiac mesenchymal cells (CMCs: $\mathrm{CD}^{-} 5^{-} \mathrm{CD} 1^{-}$), and BMDCs $\left(\mathrm{CD}^{+} 5^{+}\right.$) (Supplemental Figures 1 and 2; supplemental material available online with this article; https://doi.org/10.1172/jci.insight.131545DS1) - revealed that BMDCs constitute the majority of non-CMs in the heart 3 days after infarct ( $86.2 \%$ of live cells). By 7 days after infarct the largest cardiac cell population was CMCs (52.3\% of live cells); activated myofibroblasts likely made up a majority of this population (Figure 1, A and B, and Supplemental Figure 3A). Gene expression analysis by qPCR confirmed higher $C d h 11$ transcription in non-CMs relative to $\mathrm{CMs}$ and revealed a significant increase in non-CM Cdh11 transcription that was nearly 10-fold higher than that in sham-operated hearts (sham) by 7 days after MI (Supplemental Figure 4, A and B).

Flow cytometry further revealed that each non-CM population in the heart had some level of CDH11 expression (Figure 1, A-C) and that the overall percentage of $\mathrm{CDH} 11^{+}$cells in infarcted hearts was increased relative to sham by 7 days after infarct (18.2\% of live cells vs. $6.9 \%$ of live cells in sham; Supplemental Figure $4 \mathrm{C}$ ). Percentages of both $\mathrm{CDH} 11^{+} \mathrm{BMDCs}$ and $\mathrm{CDH} 11^{+} \mathrm{CMCs}$ increased between days 3 and 7 after MI but remained unchanged over time in sham hearts. Despite constituting less than $3 \%$ of all live cells in the heart, the percentage of $\mathrm{CDH} 11^{+} \mathrm{CECs}$ was decreased relative to sham at both 3 and 7 days after MI and changed over time in both surgical conditions (Figure $1, \mathrm{~A}-\mathrm{C}$ ). While $\mathrm{CDH} 11^{+} \mathrm{CECs}$ comprised a markedly lower percentage of all $\mathrm{CDH} 11^{+}$cells identified in infarcted hearts relative to sham at both 3 and 7 days, $\mathrm{CDH} 11^{+} \mathrm{CMCs}$ made up the majority of all $\mathrm{CDH} 11^{+}$cells after 7 days in both surgical conditions. The percentage of BMDCs expressing CDH11 constituted a significant fraction of all CDH11 ${ }^{+}$cells in the heart 3 days after $\mathrm{MI}\left(63.6 \%\right.$ of $\mathrm{CDH} 11^{+}$cells vs. $30.5 \%$ of $\mathrm{CDH} 11^{+}$cells in sham) and was reduced back to sham levels by 7 days after MI (Figure 1, A-C), consistent with the time course of inflammation resolution.

We next analyzed our flow cytometry data to determine which BMDC subsets had CDH11 expression (Figure 1, D-F). After infarct, we observed a significant increase in myeloid lineage cells $\left(C D 45^{+} \mathrm{CD} 11 \mathrm{~b}^{+}\right)$ - including neutrophils $\left(\mathrm{CD} 45^{+} \mathrm{CD} 11 \mathrm{~b}^{+} \mathrm{Gr}-1 / \mathrm{CD}^{2} 6^{\mathrm{hi}}\right)$, M1-like macrophages $\left(\mathrm{CD} 45^{+} \mathrm{CD} 11 \mathrm{~b}^{+} \mathrm{Gr}-1 /\right.$ $\left.\mathrm{CD}^{2} 6^{\text {int }} \mathrm{CD} 206^{-}\right)$, and M2-like macrophages $\left(\mathrm{CD} 45^{+} \mathrm{CD} 11 \mathrm{~b}^{+} \mathrm{Gr}-1 / \mathrm{CD} 86^{\text {int }} \mathrm{CD} 206^{+}\right)(17)$ - as well as a reduction in bone marrow-derived proangiogenic cells $\left(\mathrm{CD} 45^{+} \mathrm{CD} 11 \mathrm{~b}^{-} \mathrm{CD} 31^{+}\right)$and nonmyeloid lineage BMDCs $\left(\mathrm{CD}_{4} 5^{+} \mathrm{CD} 11 \mathrm{~b}^{-}\right)$, which are predominately lymphocytes (Supplemental Figure 3A). Though most BMDC subsets had detectable amounts of CDH11 expression, less than $1 \%$ of all Gr-1/CD86 ${ }^{\text {lo }}$ BMDCs - eosinophils and monocytes - were $\mathrm{CDH} 11^{+}$. The largest population of $\mathrm{CDH} 11^{+} \mathrm{BMDCs}$ was M2-like macrophages (Figure 1D), and the percentage of BMDCs that were $\mathrm{CDH} 11^{+} \mathrm{M} 2$-like macrophages was increased relative to sham by 7 days after $\mathrm{MI}\left(14.1 \%\right.$ of $\mathrm{CD} 45^{+}$cells vs. $9.5 \%$ of $\mathrm{CD} 45^{+}$cells in sham). Similar to $\mathrm{CECs}$, there were fewer $\mathrm{CDH} 11^{+}$bone marrow-derived proangiogenic cells in the heart at both 3 and 7 days after infarct compared with sham (Figure $1 \mathrm{~F}$ ). Though $\mathrm{CDH} 11^{+}$neutrophils comprised a relatively small percentage of all BMDCs, they represented a significant percentage of all $\mathrm{CDH} 11^{+} \mathrm{BMDCs}$ and were elevated at day 3 after $\mathrm{MI}$ relative to sham $\left(27.0 \%\right.$ of $\mathrm{CDH} 11^{+} \mathrm{CD} 45^{+}$cells vs. $10.1 \% \mathrm{CDH} 11^{+} \mathrm{CD} 45^{+}$ cells in sham). $\mathrm{CDH}_{1} 1^{+} \mathrm{M} 1$-like macrophages were also elevated at day 3 after infarct, albeit not significantly. Nonetheless, the percentages of both $\mathrm{CDH} 11^{+}$neutrophils and $\mathrm{CDH} 11^{+} \mathrm{M} 1$-like macrophages 
A $=\mathrm{CD} 45^{-} \mathrm{CD} 31^{+}-\mathrm{CD} 45^{+}$ CD45-CD31-
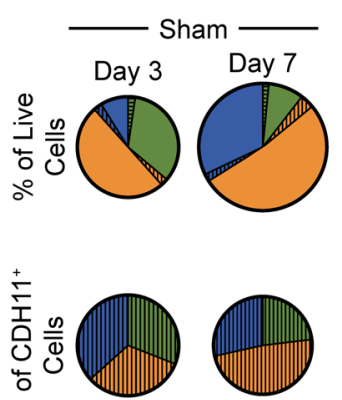

。

B
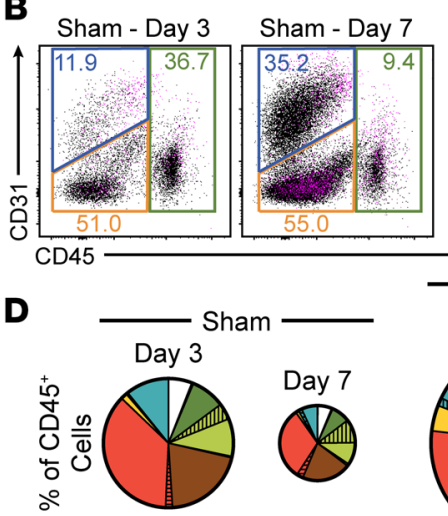

वे
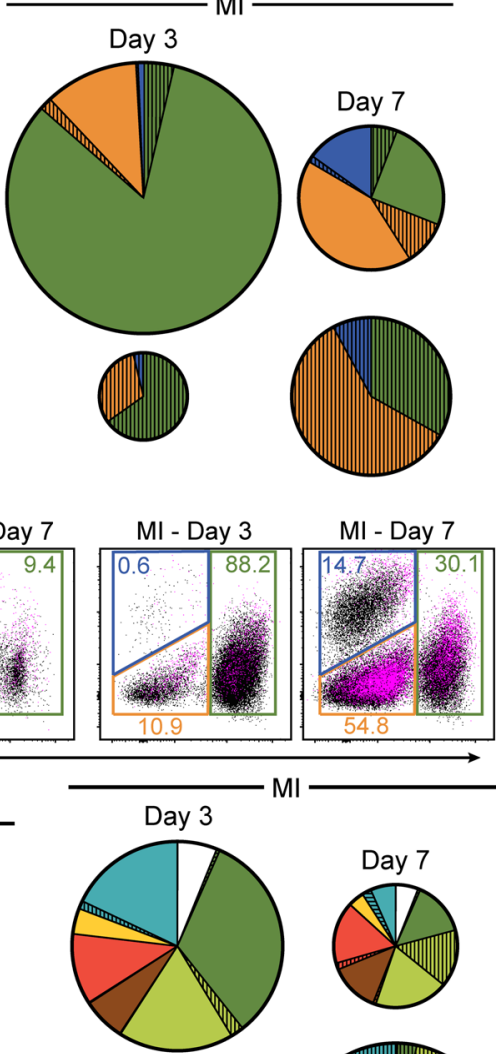

$\mathrm{Ml}$

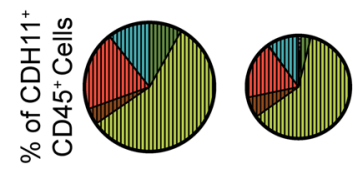

F Neutrophils M1 Macrophages M2 Macrophages BMD-PACs $\mathrm{CDH} 11^{+} \mathrm{CD} 45^{+} \mathrm{CDH} 11^{+} \mathrm{CD} 45^{+} \mathrm{CD} 11 \mathrm{~b}^{+} \mathrm{CDH} 11^{+} \mathrm{CD} 45^{+} \mathrm{CD} 11 \mathrm{~b}^{+} \mathrm{CDH} 11^{+} \mathrm{CD} 45^{\circ}$

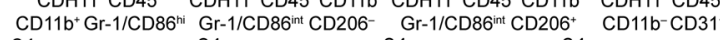
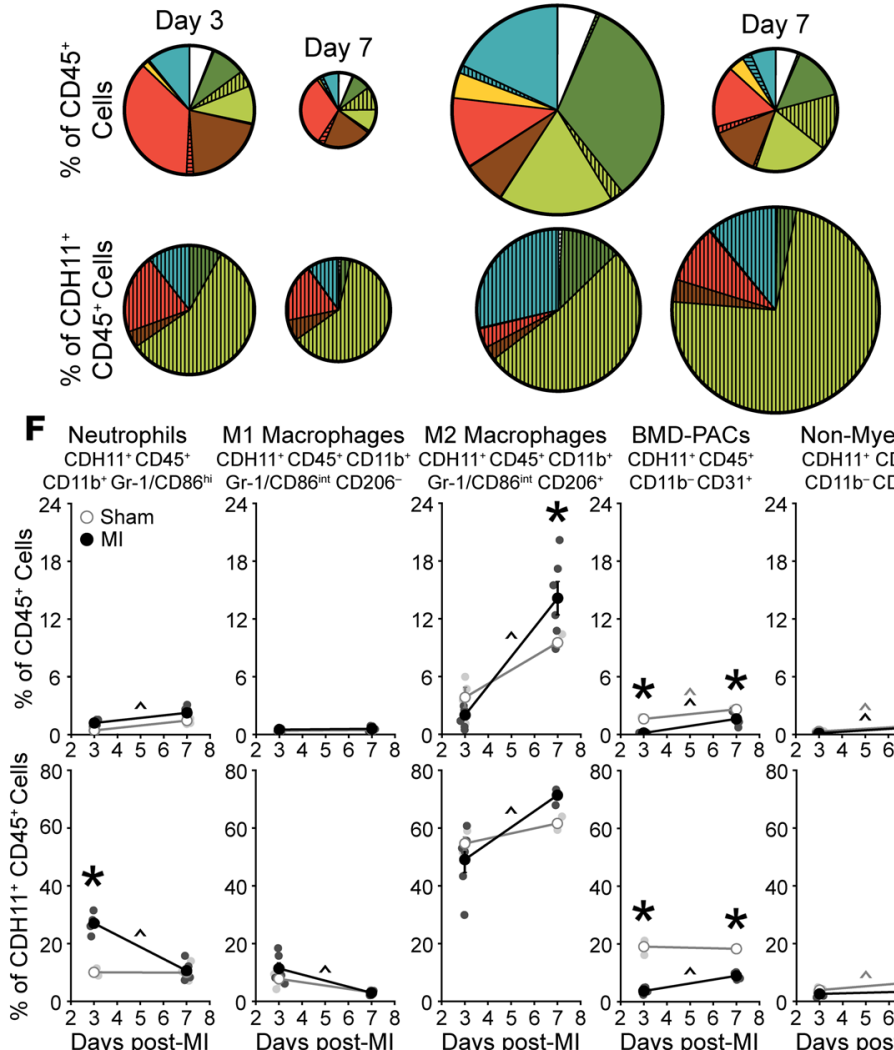

Non-Myeloid $\mathrm{CDH} 11^{+} \mathrm{CD} 45^{+}$
$\mathrm{CD} 11 \mathrm{~b}^{-} \mathrm{CD} 31^{-}$

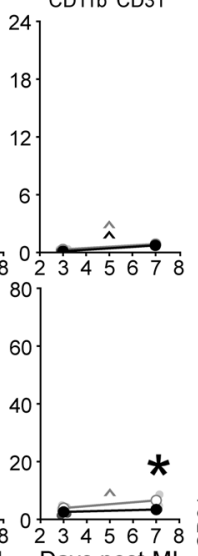

C CECs

CMCs

BMDCs

$\mathrm{CDH} 11^{+} \mathrm{CD} 45^{-} \mathrm{CD} 31^{+} \mathrm{CDH} 11^{+} \mathrm{CD} 45^{-} \mathrm{CD} 31^{-} \mathrm{CDH} 11^{+} \mathrm{CD} 45^{+}$
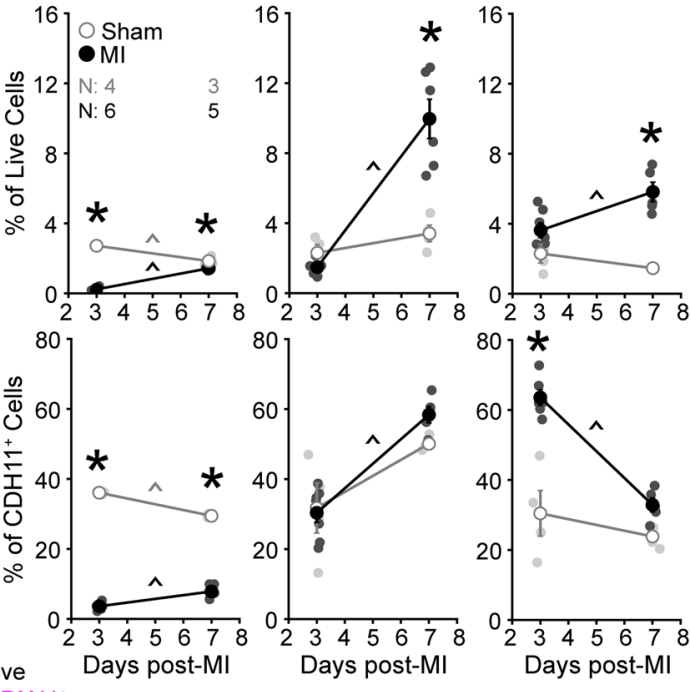

\section{CD45+CD11b+Gr-1/CD86 ${ }^{\text {hi }}$ \\ - CD45 ${ }^{+} \mathrm{CD} 11 \mathrm{~b}^{+} \mathrm{Gr}-1 / \mathrm{CD}^{\prime} 6^{\circ} \mathrm{SSC}^{\text {hi }}$ \\ - CD45+ CD11b-CD31+ \\ - $\mathrm{CD} 45^{+} \mathrm{CD} 11 \mathrm{~b}^{-} \mathrm{CD} 31^{-}$ \\ CD45+CD11 $\mathrm{b}^{+} \mathrm{Gr}-1 / \mathrm{CD} 86^{\text {int }} \mathrm{CD} 206^{+}$ \\ CD45+CD11b+Gr-1/CD86 int CD206-

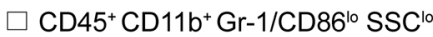 IIIII $\mathrm{CDH} 11^{+}$}

E
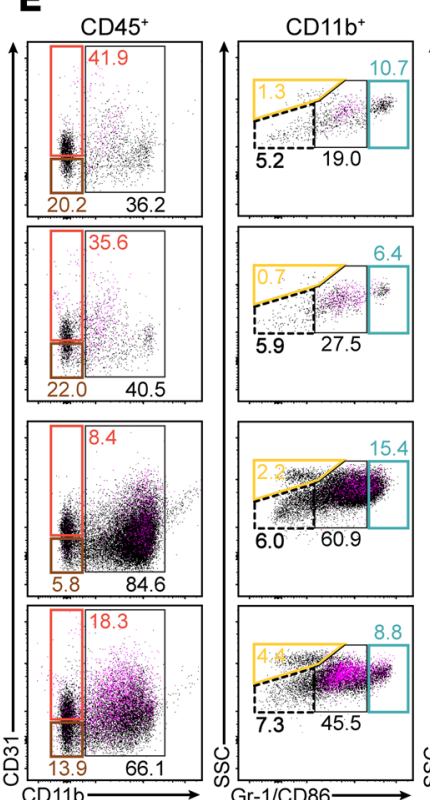

Live

$\mathrm{Gr}-1 / \mathrm{CD} 86^{\mathrm{in}}$

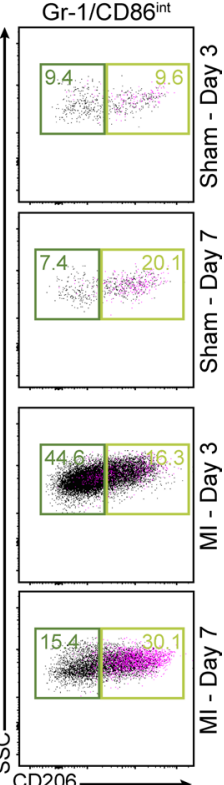

Figure 1. Specific cell populations in the heart express CDH11 after MI. Non-CM populations, including cardiac endothelial cells (CECs), cardiac mesenchymal cells (CMCs), and bone marrow-derived cells (BMDCs), express a baseline amount of CDH11 (hatched wedges) that is increased after MI. Pie chart radii (A) are scaled by either the number of live single cells (top) or the number of $\mathrm{CDH11^{+ }}$ cells (bottom) relative to sham hearts at day 3 . Representative dot plots (B) show changes in CDH11 expression (magenta) within each population (colored gates). CDH11+ cells (C) within each population are shown as either a percentage of live cells (top) or of all CDH11+ cells (bottom). CDH11 expression in BMDC (CD45+) subpopulations (D) revealed predominant expression in neutrophils and M1- and M2-like macrophages (light blue, dark green, and light green, respectively). Representative dot plots (E) show changes in CDH11 expression (magenta) within each subpopulation

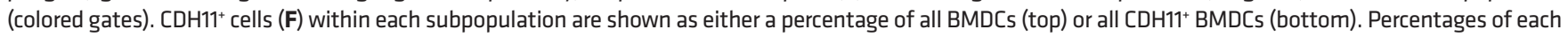
population, relative to all live cell events, are denoted within colored gates. Data are presented as mean \pm SEM, with $n=3-6$ per group; dots in $\mathbf{C}$ and $\mathbf{F}$ denote individual animals. Pie charts represent average values. Significance was determined by 2 -way ANOVA with a Holms-Sidak's multiple comparison test. ${ }^{*} P<0.05$ between sham and $\mathrm{Ml}$ at the same time, ${ }^{\wedge} P<0.05$ over time; color of significance marker denotes group. 
were decreased to sham levels by 7 days after infarct. In contrast, the percentage of all $\mathrm{CDH} 11^{+} \mathrm{BMDCs}$ that were either $\mathrm{CDH} 11^{+} \mathrm{M} 2$-like macrophages or $\mathrm{CDH} 11^{+}$bone marrow-derived proangiogenic cells increased between 3 and 7 days after infarct, whereas $\mathrm{CDH} 11^{+}$nonmyeloid cells remained unchanged after $\mathrm{MI}$ and were reduced at day 7 relative to sham (Figure 1F). A similar flow cytometric analysis profiling cells isolated from the peripheral blood after MI confirmed that the vast majority of cells ( $>90 \%$ of live cells) were $\mathrm{CD}_{4} 5^{+}$(Supplemental Figure 3B), and it revealed that only a small fraction of cells ( $<2 \%$ of live cells) expressed CDH11 (Supplemental Figure 4D). Of the $\mathrm{CDH}_{11} 1^{+}$cells in the blood, little difference was observed between sham and MI (Supplemental Figure 5).

Next, we sought to evaluate the effect of CDH11 signaling on cardiac remodeling in vivo and found that there was no difference in ejection fraction (EF) or left ventricular (LV) mass among $C d h 11^{+/+}, C d h 11^{+/-}$ , and $C d h 11^{-/-}$mice following MI (Figure 2, A and B). However, LV volume was increased in $C d h 11^{+/+}$and Cdh $11^{+/-}$mice, but not in $C d h 11^{-/-}$mice, between 7 and 21 days after infarct. By day 21, LV diastolic volume was increased in $C d h 11^{+/+}$mice relative to that in $C d h 11^{+/-}$and $C d h 11^{-/-}$mice (Figure 2, C and D), and mixed-effects analysis identified a significant difference in LV diastolic volume among genotypes (Figure 2C). In addition, genetic deletion of CDH11 appeared to provide a survival benefit in the first week after MI, with an observed incidence of $29 \%$ survival for $C d h 11^{+/+}$mice, $63 \%$ survival for $C d h 11^{+/-}$mice, and $57 \%$ survival for $C d h 11^{-/-}$mice. To further investigate the role of CDH11 in BMDCs, we induced MI in wildtype mice receiving bone marrow from $C d h 11^{+/+}, C d h 11^{+/-}$, and $C d h 11^{-/-}$donors (Figure $2 \mathrm{E}$ and Supplemental Figure 6). Though most of the mice ( 8 of 10) that received $C d h 11^{-1-}$ bone marrow died before complete bone marrow reconstitution at 6 weeks after transplantation, $C d h 11^{+/-}$bone marrow recipients showed improved EF between 7 and 21 days and reduced LV mass 7 days after MI, relative to $C d h 11^{+/+}$bone marrow recipients (Figure 2, F and G). Mixed-effects analysis identified a significant difference among genotypes and significant interaction between genotype and time for LV mass (Figure $2 \mathrm{G}$ ). LV volume was not different between mice receiving either $C d h 11^{+/+}$or $C d h 11^{+/-}$bone marrow (Figure 2, $\mathrm{H}$ and I).

Having demonstrated a role for $\mathrm{CDH} 11$ in remodeling after MI, we hypothesized that blocking CDH11 adhesion and activity may therapeutically reduce MI-induced adverse ventricular remodeling and heart failure. Thus, we treated mice with i.p. injections of either a CDH11-blocking antibody (SYN0012) or IgG2a isotype control beginning 24 hours after induction of MI. EF (Figure 3A) and LV mass (Figure 3B) were preserved in SYN0012-treated animals relative to IgG2a for up to 56 days following MI. Further, the increased LV dilation observed in the IgG2a-treated mice was curtailed in the animals receiving SYN0012, resulting in preserved ventricular volume at both diastole (Figure 3C) and systole (Figure 3D) for up to 56 days after MI. Mixed-effects analysis identified an overall significant difference between treatment groups and significant interactions between treatment and day for EF and LV volume. Though survival after MI was improved with CDH11 blockade (82\% survival for SYN0012 vs. 65\% survival for IgG2a), it was not found to be statistically significant $(P=0.20$; Supplemental Figure 7).

Given the changes in LV structure and function observed by echocardiography, we next wanted to quantify tissue mechanical properties and the extent of cardiac remodeling after infarct. In particular, median stiffness of infarcted areas from both SYN0012- and IgG2a-treated hearts, as measured by atomic force microscopy (AFM) (Supplemental Figure 8), was decreased below the measured range of sham myocardial stiffness by 7 days after MI (Figure 4A). However, by 21 days, infarct stiffness from both treatment groups was increased relative to sham, with the median and range of stiffness values from IgG2a-treated infarcts exceeding that of time-matched SYN0012-treated infarcts. By 56 days after MI, the median stiffness of SYN0012-treated infarcts had returned to the sham stiffness range, while the stiffness of IgG2a-treated infarcts remained elevated (Figure 4A). Automated histological quantification (Supplemental Figure 9) determined that infarcts from SYN0012-treated animals were thicker and spanned less of the LV circumference than IgG2a at both 21 and 56 days after MI (Figure 4, B-D, and Supplemental Figure 10, D and E). There were no measured differences in cardiac hypertrophy or remote interstitial fibrosis between treatments (Supplemental Figure 10, A-C), and despite differences in infarct remodeling, we observed no difference in CF contractility following SYN0012 treatment in vitro. Whereas Cdh11-knockout reduced collagen gel contraction by CFs (Supplemental Figure 11A), SYN0012 treatment did not affect CF contraction (Supplemental Figure 11B), suggesting a potentially critical role for $\mathrm{CDH} 11$ activation in modulating the myofibroblast phenotype and promoting interactions with specific immune cell populations in order to determine the extent of cardiac remodeling after infarct. 

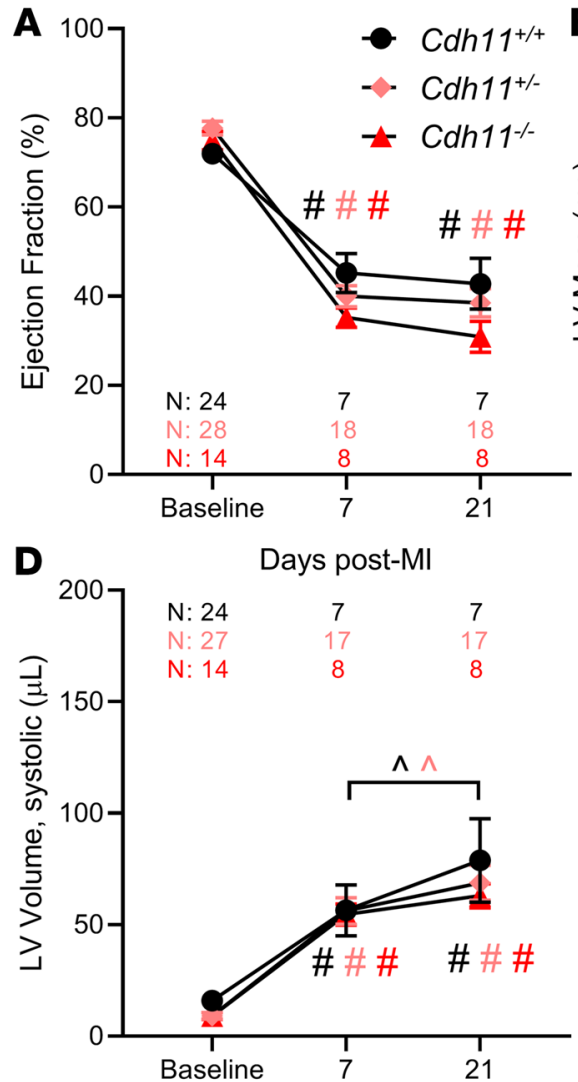

F $100 \quad$ Days post-Ml

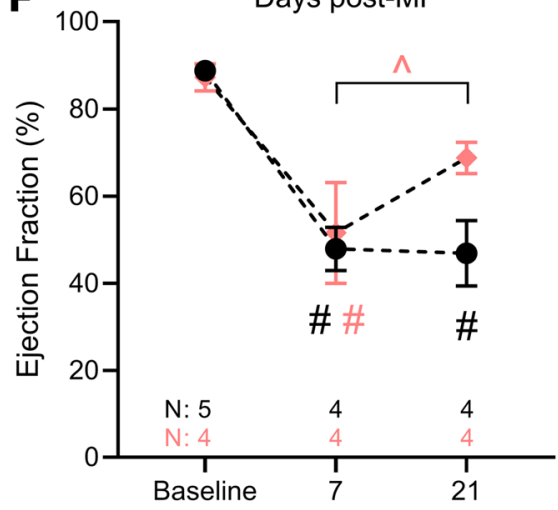

Days post-MI

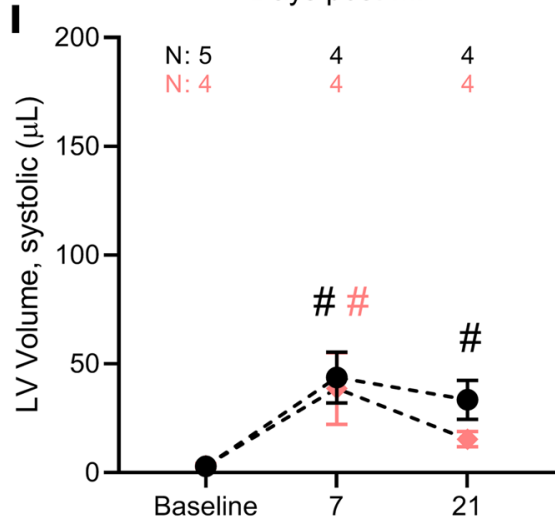

Days post-MI

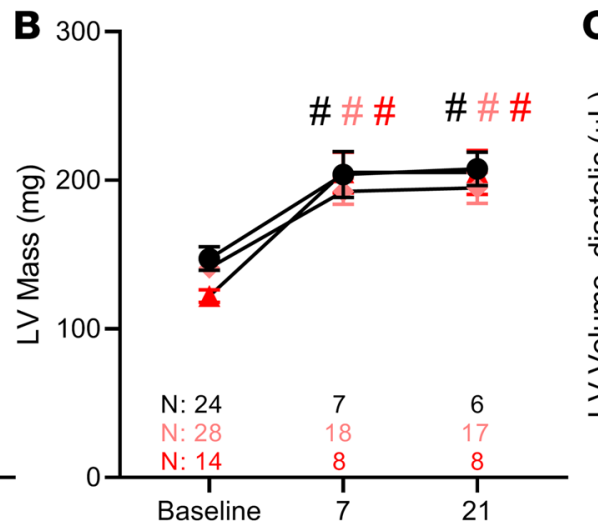

E

Days post-MI

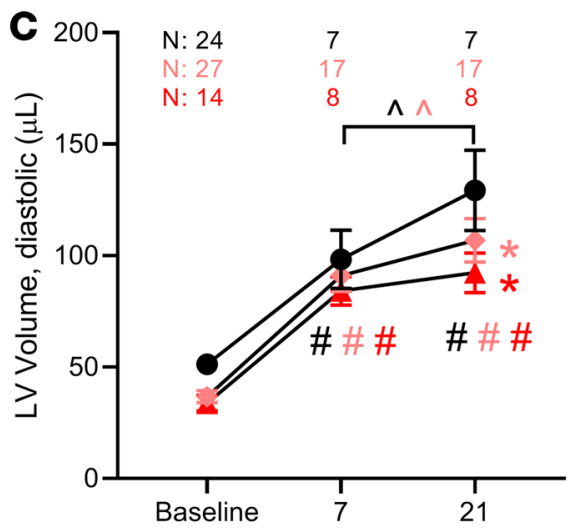

Days post-MI

\section{WT (C57BL6/J) irradiated Bone marrow $\quad$ Ml by LAD 6 weeks transplant

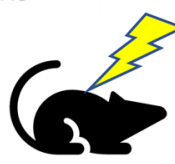

Cdh $11^{+/+}$

Cdh11+s

Cdh11\%

6 weeks for bone

marrow reconstitution

G
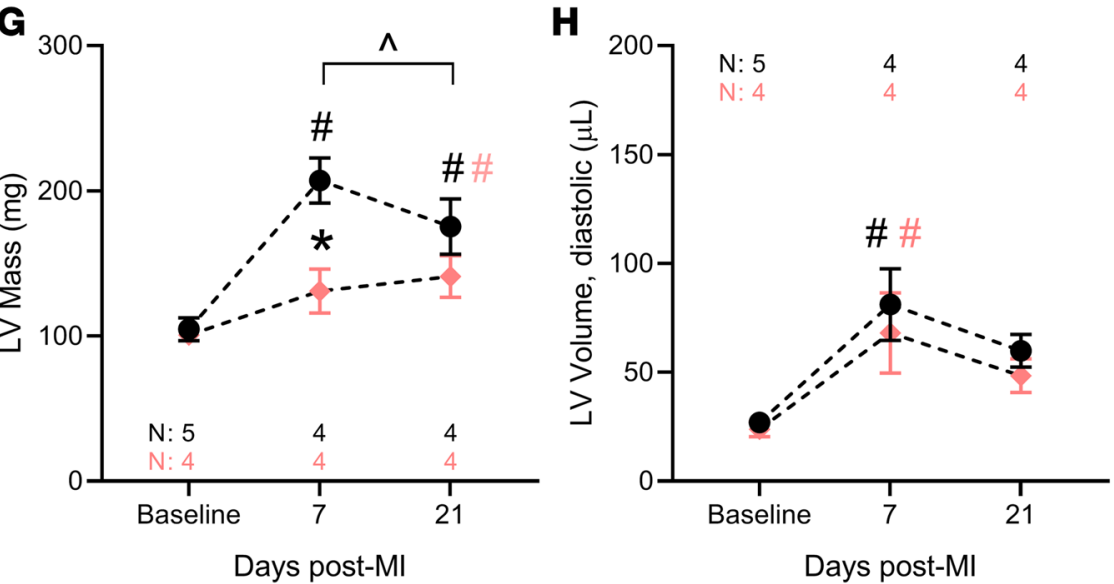

BMT donor:

Figure 2. CDH11 mediates remodeling after $\mathrm{MI}$ in a partially bone marrowderived cell-dependent manner. Echocardiographic analysis of infarcted hearts revealed that ejection fraction (A), left ventricular (LV) mass (B), and LV volume (C and $\mathbf{D})$ were significantly changed from baseline in all groups (A-D). In addition, Cdh11+/- and $C d h 11^{-/-}$mice had significantly reduced LV mass and diastolic volume

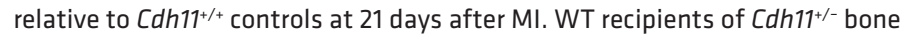
marrow (E) had significantly higher EF at 21 days than at 7 days $(\mathbf{F})$ and significantly lower LV mass at 7 days after $\mathrm{MI}$ (G) than age-matched $\mathrm{Cdh} 11^{+/+}$bone marrow recipients. There was little difference in $\mathrm{LV}$ volume $(\mathbf{H}$ and $\mathbf{I})$ between donor groups. Data are presented as mean \pm SEM. Significance was determined by mixed-effect analysis, with a Holms-Sidak's multiple comparison test. ${ }^{*} P<0.05$ relative to $C d h 11^{+/+},{ }^{\sharp} P<0.05$ relative to baseline, ${ }^{\wedge} P<0.05$ between time points; color of significance marker denotes group. 

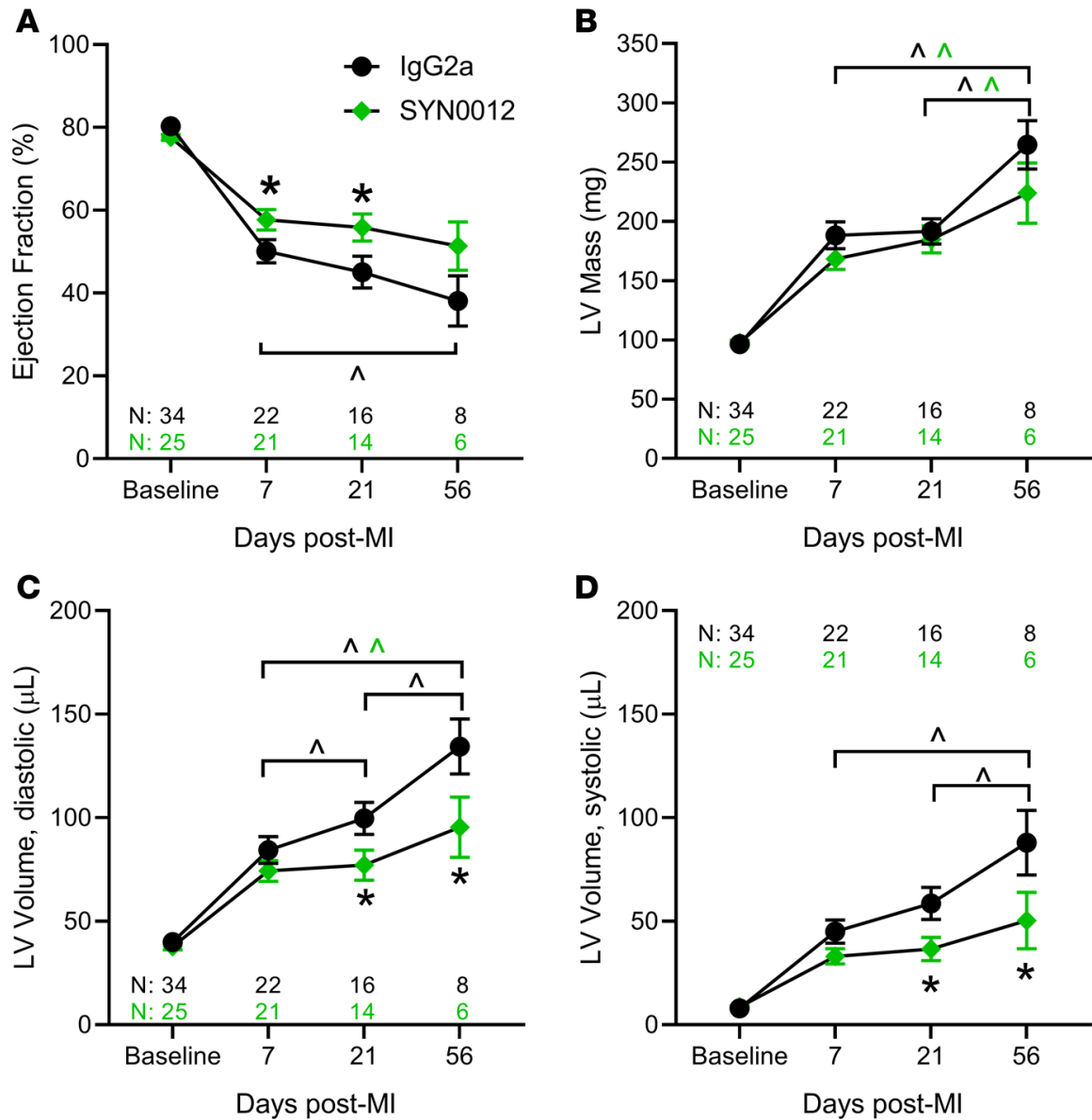

Figure 3. CDH11 blockade improves functional outcomes after MI. Echocardiographic analysis of infarcted hearts revealed that ejection fraction (A), left ventricular (LV) mass (B), and LV volume (C and $\mathbf{D})$ was significantly changed from baseline in all groups. SYN0012-treated hearts had significantly higher ejection fraction at 7 and 21 days after infarct, and reduced LV volume at 21 and 56 days after infarct, compared with IgG2a-treated controls; LV mass was not different between treatments at any time point. Data are presented as mean $\pm \mathrm{SEM}$. Significance was determined by mixed-effect analysis with a Holms-Sidak's multiple comparison test. ${ }^{*} P<0.05$ between treatments, ${ }^{\wedge} P$ $<0.05$ between time points; color of significance marker denotes treatment group.

To determine which cells contribute to the preserved cardiac remodeling in response to pharmacologic $\mathrm{CDH} 11$ blockade, we again used flow cytometry to quantify the percentages of non-CMs in the heart and peripheral blood after infarct (Figure 5). SYN0012 treatment did not alter the percentages of the primary non$\mathrm{CM}$ cardiac cell populations - $\mathrm{CECs}, \mathrm{CMCs}$, and $\mathrm{BMDCs}$ - relative to IgG2a treatment, despite trends toward decreased CMCs and increased BMDCs after infarct in SYN0012-treated hearts (Figure 5, A-C). Further examination of BMDC subsets revealed that SYN0012 treatment decreased the percentage of specific myeloid lineage cells - particularly, neutrophils and M1-like macrophages - in the heart at 3 days after MI (Figure 5, D-F). Though the ratio of M1/M2-like macrophages in the heart was not altered with SYN0012 treatment (Supplemental Figure 12, A-C), the early reduction in neutrophils and M1-like macrophages suggests that CDH11 blockade alters the expression of cell populations in the heart to result in a more proresolving inflammatory environment after infarct. SYN0012 also resulted in an increase in bone marrow-derived proangiogenic cells and nonmyeloid BMDCs in the heart 3 days after infarct; differences relative to IgG2a in all populations were gone by day 7 , consistent with the time course of inflammation resolution. The distribution of circulating cells in the peripheral blood was largely unaffected by SYN0012 treatment, aside from a higher M1/M2-like macrophage ratio in the peripheral blood at day 3 after infarct, relative to IgG2a (Supplemental Figure 12F). The observed increase was a result of decreased circulating levels of M2-like macrophages and suggests that SYN0012 treatment may alter macrophage recruitment to the heart after infarct (Supplemental Figure 13, D-F).

We next examined transcriptional levels of multiple inflammatory and fibrotic genes over the 21-day time course of remodeling after MI (Figure 6 and Supplemental Figure 14). SYN0012 treatment decreased 
A

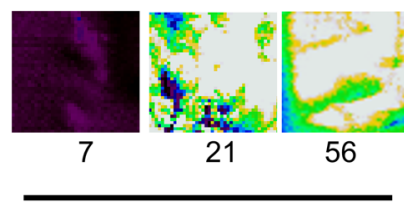

$\lg \mathrm{G} 2 \mathrm{a}$

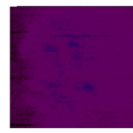

7

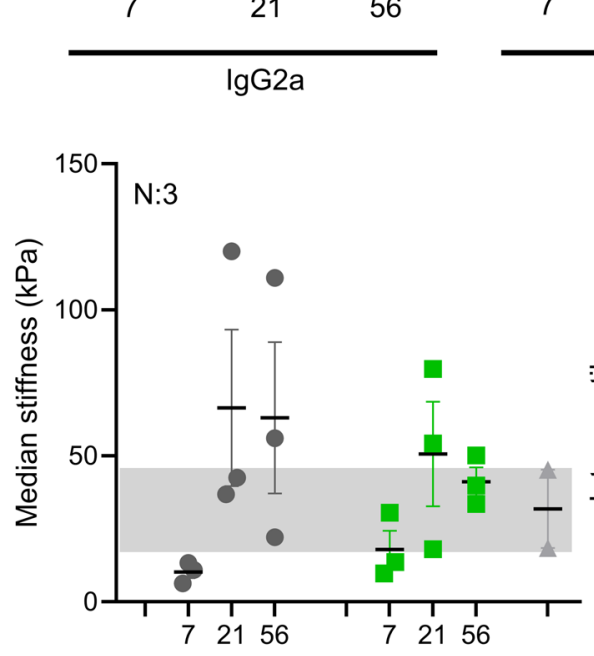

72156

Days post-M

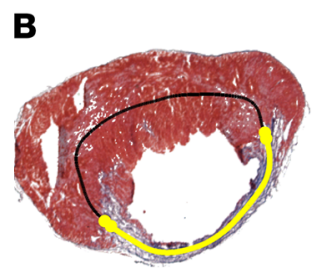

$\lg \mathrm{g} 2 \mathrm{a}$

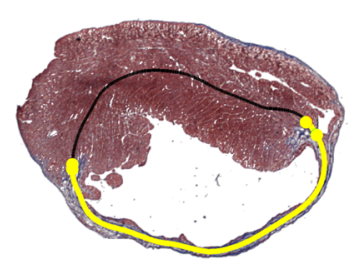

$\lg \mathrm{g} 2 \mathrm{a}$
$100 \mathrm{kPa}$

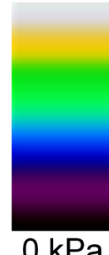

SYN0012

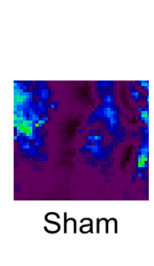

$0 \mathrm{kPa}$

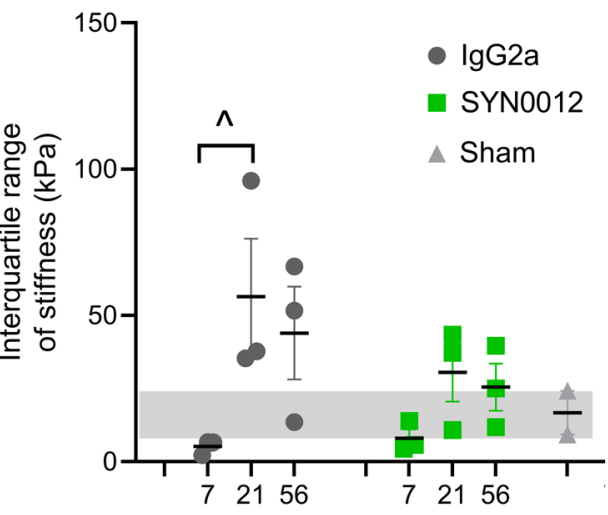

Days post-MI
SYN0012

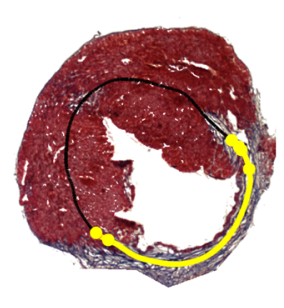

Day 21

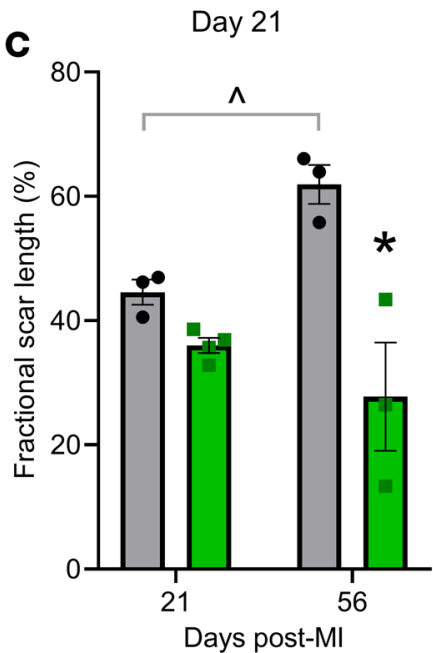

Day 56

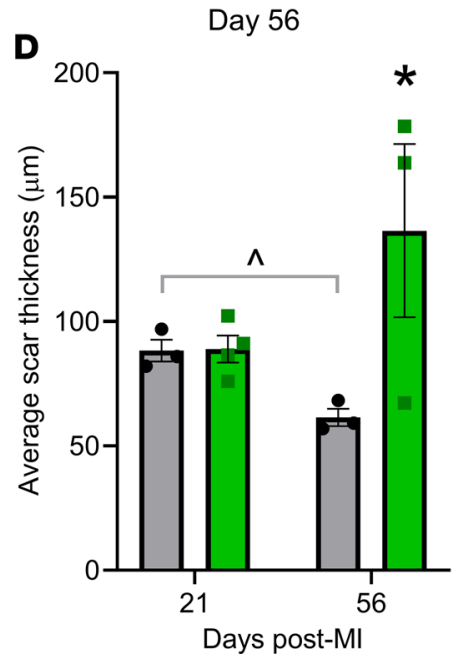

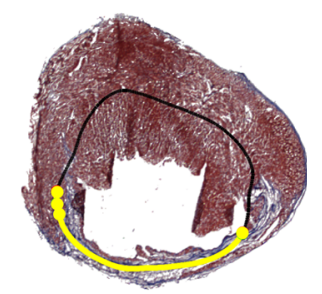

SYN0012
Figure 4. CDH11 blockade limits fibrotic remodeling after MI. Atomic force microscopy was used to quantify local tissue stiffness, as shown by representative stiffness color maps (A). Calculation of median stiffness values from each scan revealed a decrease at 7 days after MI, with all of the IgG2a hearts falling outside the range of the sham myocardium. Median stiffness values increased by 21 days and remained elevated out to 56 days in lgG2a-treated hearts, whereas SYN0012-treated hearts increased to a lesser extent at 21 days and were restored to sham levels by 56 days after $\mathrm{Ml}$; the interquartile range of stiffness values was larger in IgC2a-treated infarcts than SYN0012-treated infarcts, which were similar to the range of sham myocardium. Representative Masson's trichrome-stained sections denote scar location (yellow line), as identified by a custom image processing algorithm (B). Images were used to quantify the fractional scar length as a percentage of cardiac circumference (C) and the average thickness along the infarct length (D). Data are presented as mean $\pm \mathrm{SEM}$; each dot represents the average of either 5 independent measurements (A) or 3 independent images (C and D) from individual animals, with $n=3-4$. Significance was determined by 2-way ANOVA with Holm-Sidak's multiple comparison test. ${ }^{*} P<0.05$ between treatments, ${ }^{\wedge} P<0.05$ between time points

transcription of the proinflammatory cytokine Il6 at 3 days after MI, relative to IgG2a treatment (Figure 6A). Representative immunostaining further confirmed that there was a reduction in IL- 6 expression in the infarct region of SYN0012-treated hearts ( $~ 80 \%$ of staining intensity of sham; Figure $6 \mathrm{~B})$. Transcription of $C d h 11$ was increased at both 7 and 21 days after MI, relative to sham but was not significantly affected by SYN0012 treatment (Figure 6C). Interestingly, SYN0012 treatment reduced transcription of proangiogenic signaling 

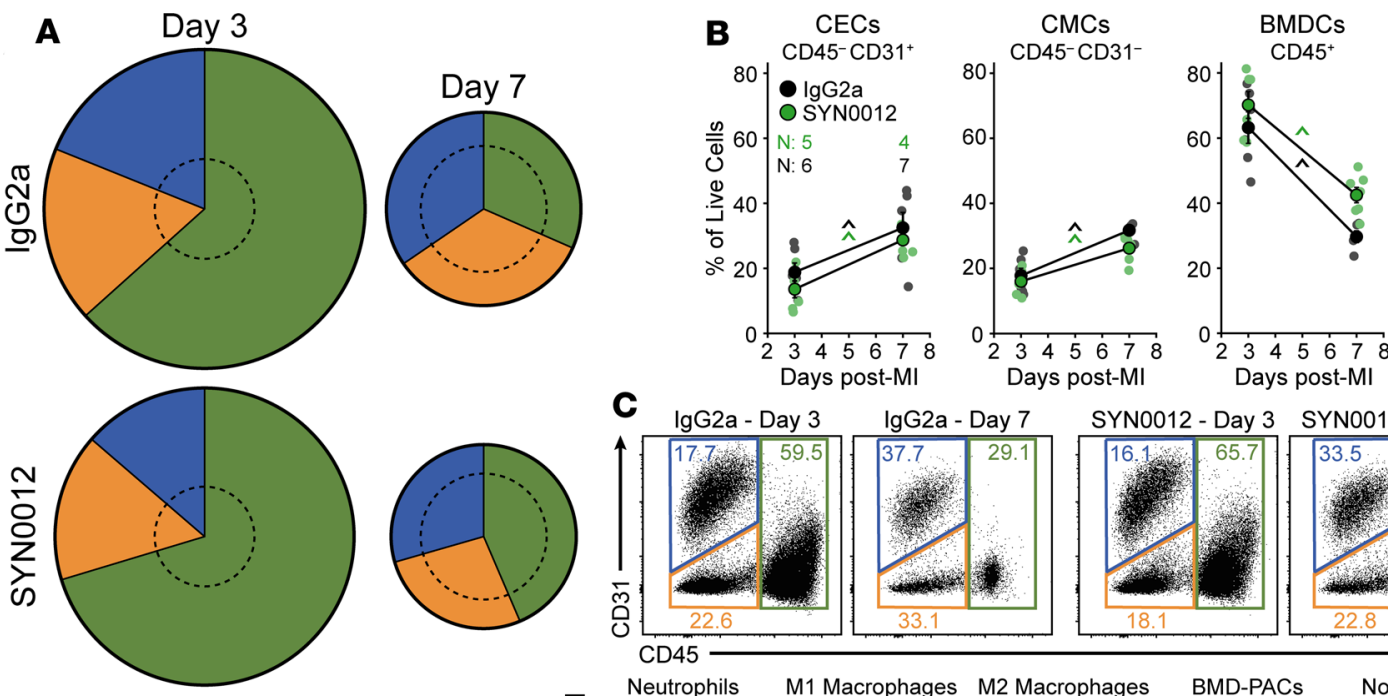

C IgG2a-Day $3 \quad \lg 2 a-$ Day 7
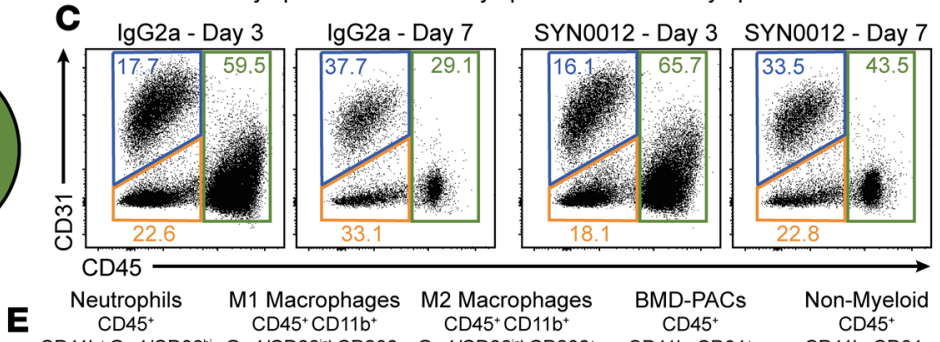

- CD45-CD31

CD45-CD31

CD45
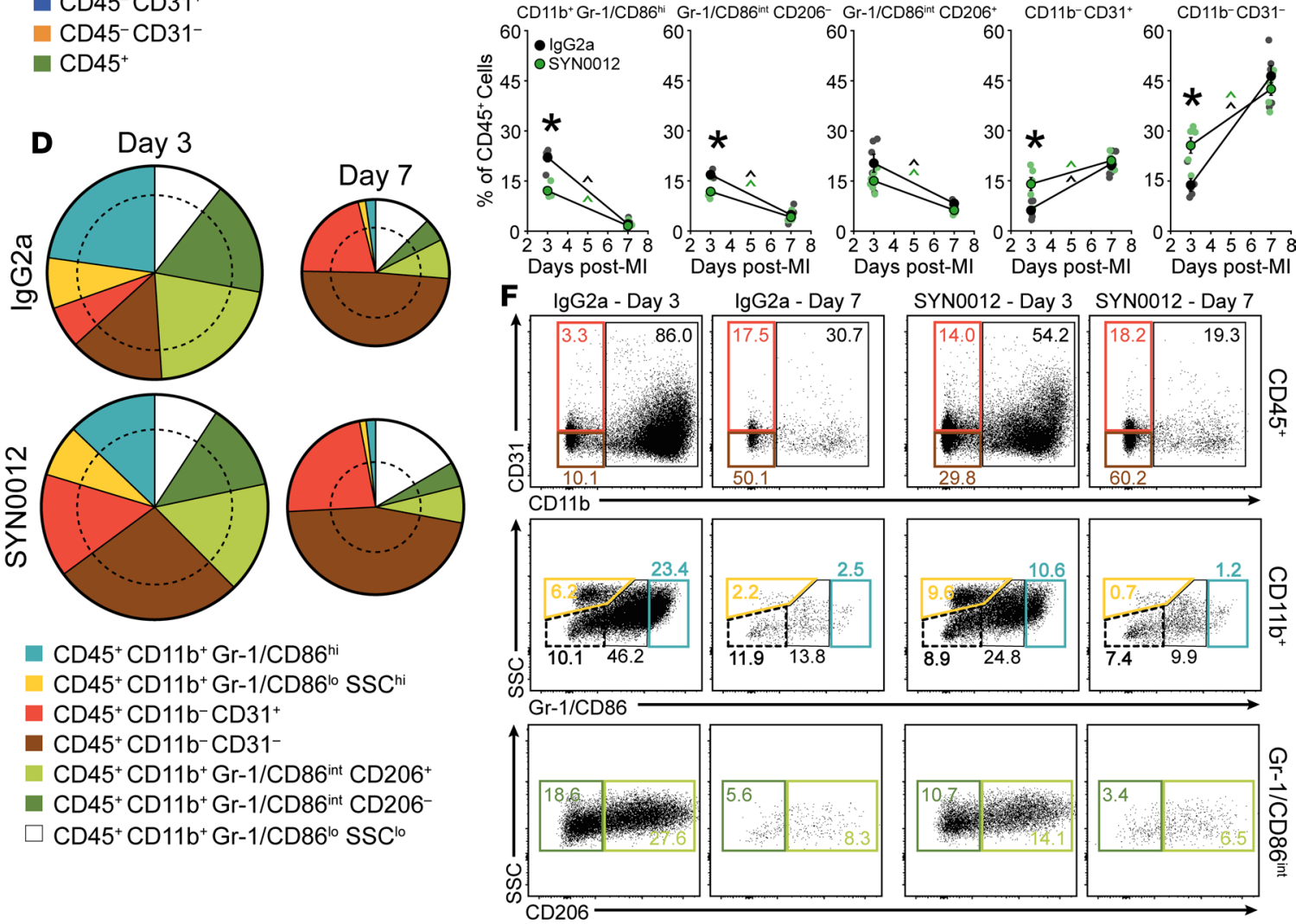

Figure 5. CDH11 blockade modulates expression of specific cell populations after MI. CDH11 blockade by SYN0012 treatment does not significantly alter the percentages of non-CM populations (cardiac endothelial cells [CECs], cardiac mesenchymal cells [CMCs], and bone marrow-derived cells [BMDCs]) in the heart after MI, relative to IgG2a isotype control (A and B). Pie chart radii are scaled by the number of live single cells for each treatment and time, relative to sham hearts at day 3 (denoted by dotted circles). Representative dot plots (C) show changes in expression of each population (colored gates). Separation of BMDC populations (D) revealed that SYN0012 treatment results in a significant reduction in neutrophils (light blue) and M1-like macrophages (dark green) in addition to increased bone marrow-derived proangiogenic cells (BMD-PACs, red) and nonmyeloid BMDCs (or lymphocytes, brown) at day 3 after infarct; differences in all populations between treatments were gone by day 7 (E). Representative dot plots (F) show changes in expression of each subpopulation (colored gates). Percentages of each population, relative to all live cell events, are denoted within colored gates. Data are presented as mean \pm SEM, with $n=4-7$ per group; dots in $\mathbf{B}$ and $\mathbf{E}$ denote individual animals. Pie charts represent average values. Significance was determined by 2 -way ANOVA with a Holms-Sidak's multiple comparison test. ${ }^{*} P<0.05$ between treatments at the same time, ${ }^{\wedge} P<0.05$ over time; color of significance marker denotes treatment group 
factors Fgf2 and Vegfal, relative to IgG2a. In particular, IgG2a-treated hearts had increased Fgf2 transcription, relative to sham, at 7 and 21 days after MI (Figure 6D). While Vegfa1 transcription was not different from sham at any time point, SYN0012 treatment reduced transcription at 7 and 21 day after infarct, relative to IgG2a (Figure 6E); however, SYN0012-treated animals appeared to have more muscularized vessels likely arterioles — in the infarct region at 21 days after MI (Figure $6 \mathrm{~F})$. Smooth muscle $\alpha$-actin ( $\alpha$ SMA) is a contractile form of actin expressed in both myofibroblasts and smooth muscle cells, which surround arteries and arterioles. Albeit not statistically significant, SYN0012 treatment resulted in an approximately 14\% reduction in the expression of $\alpha \mathrm{SMA}^{+}$myofibroblasts in infarcted regions, relative to IgG2a.

Based on our findings of increased CDH11 expression in bone marrow-derived macrophages and resident CMCs (which we expect to be primarily CFs), we next wanted to determine if interactions between these cell populations might regulate the expression or transcription of proinflammatory, profibrotic, or proangiogenic factors (Figure 7). Indeed, in vitro $\mathrm{CF}$-macrophage cocultures with varying $\mathrm{CF} /$ macrophage ratios confirmed previously reported findings that $\mathrm{CDH} 11$-dependent interactions between CFs and macrophages promote IL-6 secretion by CFs (ref. 18 and Figure 7A). Of note, macrophages alone expressed very low levels of IL-6, suggesting that CFs are the main source of IL-6 in CF-macrophage cocultures. Treatment with SYN0012 reduced, but did not prevent, secretion of IL-6 by CFs (Figure 7B). Gene expression analysis by qPCR further revealed that $M m p 13$ transcription was increased in a manner dependent upon the $\mathrm{CF} /$ macrophage ratio but was not significantly affected by SYN0012 treatment, despite a slight increase relative to IgG2a in the 50:50 culture condition (Supplemental Figure 16, A and B). Tgfß1 transcription was also increased by CF-macrophage interactions in a similar manner to Mmp13 (Supplemental Figure 16C), but transcription of proangiogenic factors Fgf2 and Vegfa1 was not affected by varying CF/macrophage ratios (Supplemental Figure 16, D and E). Transcription of macrophage polarization markers Cd14 and Mrcl was unaffected by SYN0012 treatment, whereas expression of Arg1 - a marker of M2 macrophage polarization - was increased relative to IgG2a treatment in the 50:50 culture condition (Supplemental Figure 16, F and G).

\section{Discussion}

Our findings reveal that CDH11 is expressed primarily in non-CMs of the ischemic heart and suggest a functional role for $\mathrm{CDH} 11$ in resolving tissue breakdown and promoting myocardial remodeling. Cdh11 transcription is nearly 10-fold higher at 7 days after MI, as compared with sham (Supplemental Figure 4, A and B), suggesting a prominent role in myofibroblasts, which are particularly active when the bulk of scar formation occurs between 2 and 3 weeks after MI (3). However, the observation that Cdh11 is also significantly upregulated as early as 3 days after infarct suggests that other cell types - particularly those derived from infiltrating inflammatory cells, such as neutrophils, monocytes, and macrophages - may also contribute to CDH11-mediated cardiac remodeling. Flow cytometric analysis confirmed that CDH11 was expressed in the ischemic heart and further revealed that there was baseline $\mathrm{CDH} 11$ expression in approximately $5 \%$ of all non-CMs (Supplemental Figure 4C), distributed evenly among CECs, CMCs, and BMDCs. Three days after MI, the majority of $\mathrm{CDH}_{1} 1^{+}$cells were BMDCs, whereas by day 7, the majority of $\mathrm{CDH} 11^{+}$cells were $\mathrm{CMCs}$, consistent with the time course of inflammation resolution and myofibroblast activation (Figure 1, A-C). Note that increased CDH11 expression is known to be a hallmark of myofibroblast phenotype in other cardiovascular cell types, such as aortic valve interstitial cells (19). At both 3 and 7 days after MI, the majority of $\mathrm{CDH} 11^{+} \mathrm{BMDCs}$ were myeloid lineage neutrophils and macrophages with either M1-like or M2-like polarization. At each time point, the highest expression of $\mathrm{CDH} 11$ was found in M2-like macrophages (Figure 1, D-F), consistent with prior reports of CDH11 expression in activated macrophages (20). The time-dependent expression of CDH11 in the ischemic heart suggests that targeting of CDH11 after MI has the potential to mitigate both early neutrophiland macrophage-mediated inflammation and tissue breakdown and later myofibroblast-mediated collagen deposition and remodeling to result in substantial functional improvement in the heart after infarct.

When evaluating the effect of $\mathrm{CDH} 11$ on cardiac remodeling in vivo, we found that $C d h 11^{-1-}$ mice did not have significantly altered EF after MI when compared with $C d h 11^{+/+}$controls (Figure $2 \mathrm{~A}$ ). While LV volume continued to increase in $C d h 11^{+/+}$and $C d h 11^{+/-}$mice between day 7 and 21, the LV dimensions of Cdh $11^{-1-}$ animals did not change significantly over this period, suggesting that CDH11 contributes to the fibrotic remodeling phase of MI healing (Figure 2, B-D). A larger percentage of $C d h 11^{+/-}$and $C d h 11^{-1-}$ animals survived the first week after $\mathrm{MI}$ than $\mathrm{Cdh11^{+/+ }}$ animals, suggesting that the presence of $\mathrm{CDH} 11$ in the immune cells present during the initial inflammatory phase of healing may contribute to the negative remodeling that leads to cardiac rupture in mice. Transplantation of bone marrow from $C d h 11^{-1-}$ donors 

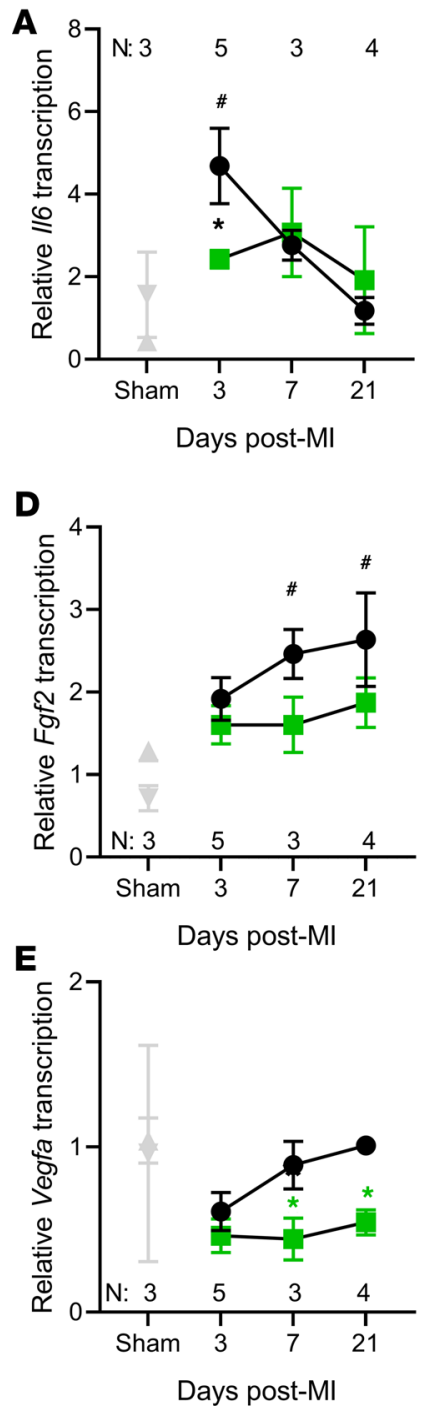

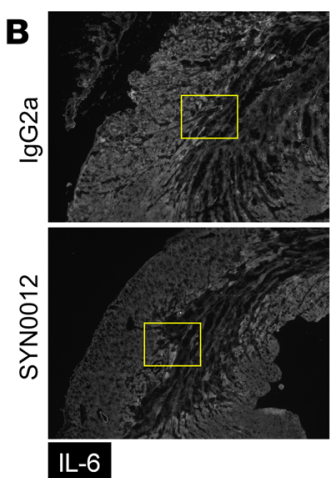

IL-6

$\mathbf{F}$
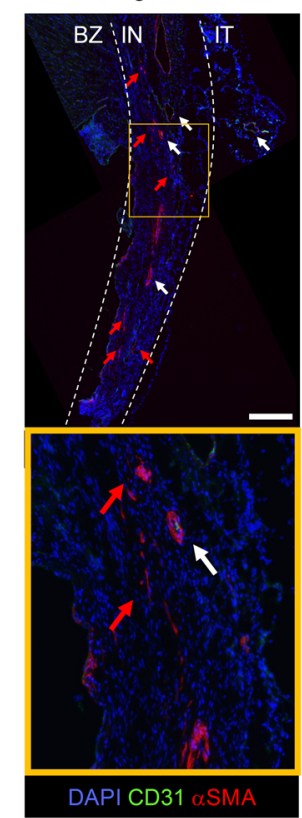

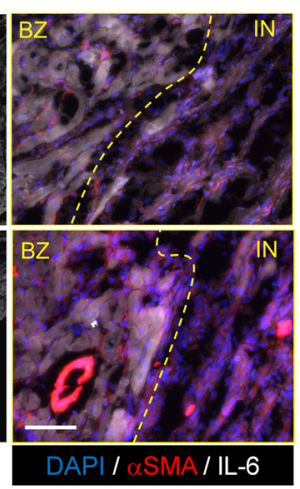

SYN0012
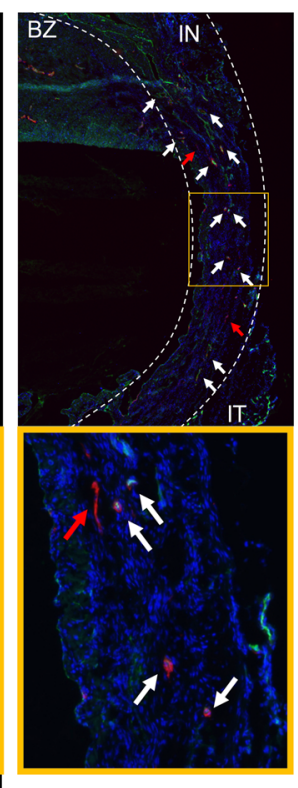
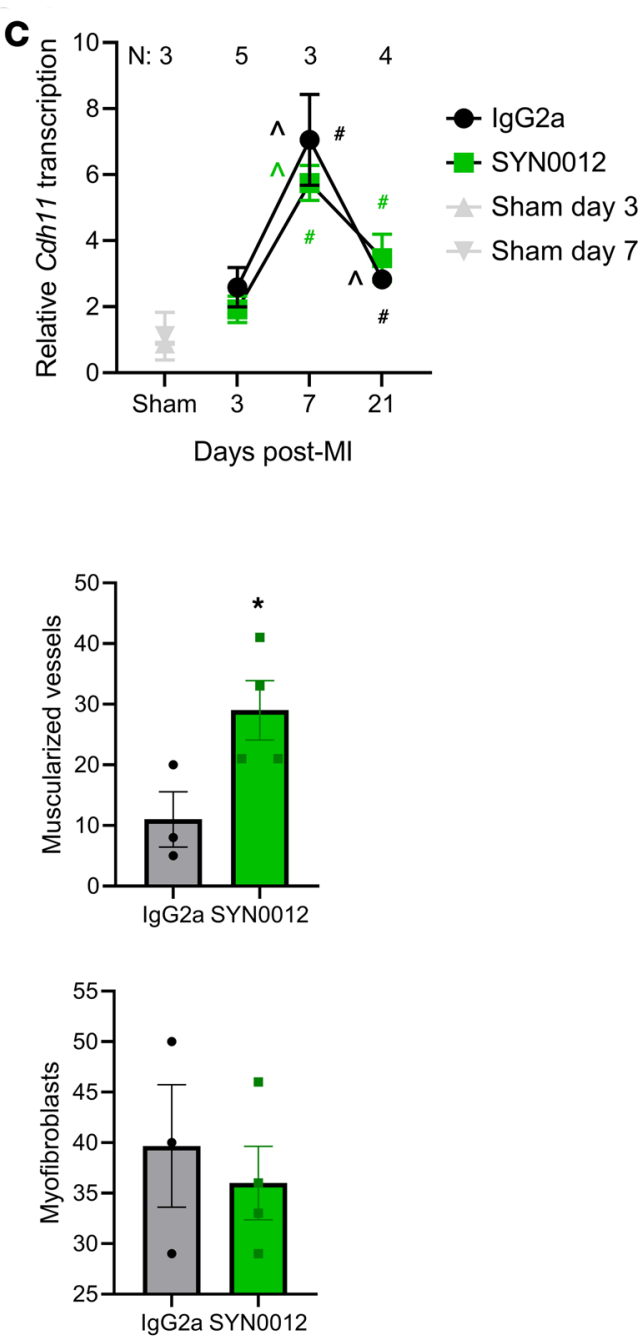

Figure 6. CDH11 blockade reduces inflammatory signaling and improves vascular maturity after MI. SYN0012 treatment significantly decreased transcription of $/ / 6$ (A) at 3 days after MI, relative to IgC2a. Immunostaining of infarct regions of hearts 3 days after MI showed decreased IL-6 expression 3 days after MI, primarily in the non-CMs - costained with $\alpha$ SMA and CD45 (cf. Supplemental Figure 15) of the infarct (B). Transcription of Cdh11 (C) was significantly increased above sham at days 7 and 21 in both treatment groups. Transcription of both Fgf2 (D) and Vegfa1 (E) was decreased by SYN0012, relative to IgC2a. Analysis of histological sections showed a significant increase in the number of muscularized vessels found in the infarct (IN) region of SYN0012-treated animals, but no difference in the number of myofibroblasts per field of view (F). Vessels in the border zone (BZ) and epicardial inflammatory tissue (IT) were not counted. Red arrows indicate myofibroblasts, white arrows indicate arterioles, and the yellow box is magnified below. Scale bar: $100 \mu \mathrm{m}$ (B); $500 \mu \mathrm{m}$ (F). Data are presented as mean \pm SEM; dots in $\mathbf{F}$ represent individual animals. Significance was determined by 2-way ANOVA analysis with a Holms-Sidak's multiple comparison test (A-E) or a 2-tailed Student's $t$ test $(\mathbf{F}) .{ }^{*} P<0.05$ between treatments, ${ }^{*} P<0.05$ relative to sham,

$\wedge P<0.05$ between time points; color of significance marker denotes treatment group.

was fatal to most of the WT recipients, likely due to impaired localization of hematopoietic cells to the bone marrow niche (ref. 12 and Supplemental Figure 6). However, surviving $C d h 11^{+/}$bone marrow recipients had improved EF 3 weeks after MI and reduced LV mass, relative to $C d h 11^{+/+}$bone marrow recipients, 1 week after MI (Figure 2, F-I). It is interesting to note that the cohort that received bone marrow transplants had less severe responses to MI, which might be due to the inherent effects of irradiation and bone marrow transplantation on modulating the expression of macrophage populations and after MI remodeling (21). Overall, these data suggest that CDH11 has important functional roles in multiple resident and recruited cell types, including the migration and localization of BMDCs and the activity of myofibroblasts. Note that $C d h 11^{-1-}$ aortic valve interstitial cells have reduced contractility (19), which we also observed in Cdh $11^{-1-}$ CFs but notably did not observe in wild-type CFs treated acutely with SYN0012 (Supplemental Figure 11). This defect in CF contractility and other reported effects of the permanent global knockout of 


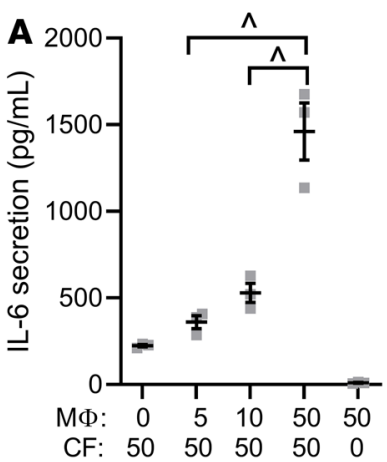

Thousands of cells

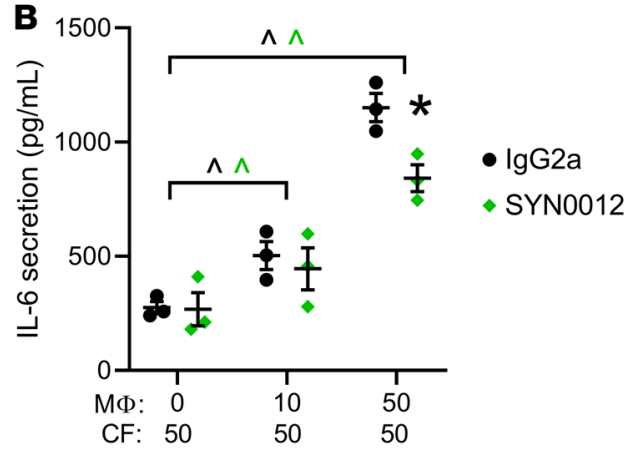

Thousands of cells

Figure 7. Coculture of macrophages and cardiac fibroblasts increases proinflammatory and profibrotic signaling. Coculture of macrophages and cardiac fibroblasts (CFs) promotes secretion of IL-6 by CFs in a macrophage-dependent manner (A). CDH11 blockade by SYN0012 treatment significantly reduced IL-6 secretion in CF-macrophage cocultures at high macrophage concentrations (B). Secreted IL-6 was measured in media of CF-macrophage cocultures by ELISA. Data are presented as mean \pm SEM; each dot represents a biological replicate $(n=3)$. Significance was determined by 1- and 2-way ANOVA, with a Holms-Sidak's multiple comparison tests. ${ }^{\wedge} P<0.05$ between macrophage conditions, ${ }^{\#} P<$ 0.05 relative to CF-only control, ${ }^{*} P<0.05$ between treatments; color of significance marker denotes treatment group.

CDH11 $(22,23)$ in the native cells of the heart are likely the reason we did not see the same improvement in $\mathrm{EF}$ and changes in LV volume after $\mathrm{MI}$ in the genetic model as in the bone-marrow recipient mice or acute SYN0012-treated animals.

Previous studies have shown that genetic and pharmacologic targeting of CDH11 improves functional responses in both fibrotic lung disease and aortic valve calcification (10, 11). Herein, CDH11 blockade by SYN0012 treatment appears to preserve cardiac function as early as 7 days after MI and prevents the continued functional decline observed with IgG2a treatment out to 56 days (Figure 3A). LV volume was not different at 7 days, but the dramatic increases in both diastolic and systolic volume between 7 and 56 days in IgG2a-treated animals were prevented by pharmacological inhibition of CDH11 by SYN0012 (Figure 3, C and D). This observation, combined with increased Cdh11 transcription occurring at day 7 after MI, suggests that CDH11-expressing cells play an active role in LV remodeling and dilation during the fibrotic phase of infarct healing. Albeit not statistically significant, twice as many IgG2a-treated mice died between days 3 and 7 after MI - the highest risk time period for cardiac rupture (3) - than in the SYN0012-treated cohort (Supplemental Figure 7). Indeed, SYN0012-treated mice also showed increased survival rates relative to prior reports using the same surgical model of MI (24). This difference in mortality is likely a consequence of altered tissue breakdown and inflammation-mediated softening of the myocardial wall, consistent with the reduction in stiffness observed by AFM measurements at day 7 after MI; by day 21, and even more so at day 56, we observed high stiffness variability in IgG2a-treated infarcts, whereas SYN0012-treated infarcts had more consistent stiffness values within the range of sham animals (Figure 4).

Having identified a clear functional effect of CDH11 blockade in limiting myocardial remodeling and infarct expansion after MI, we investigated which cell types and molecular mechanisms may mediate this process. Flow cytometry-based assessment of primary non-CM populations in the ischemic heart revealed that SYN0012 treatment did not affect the overall percentages of CMCs or BMDCs at 3 or 7 days after MI (Figure 5, A-C). However, further analysis of specific BMDC subpopulations revealed that targeting CDH11 alters the early expression of myeloid lineage neutrophils and macrophages, shifting the balance from proinflammatory macrophages and neutrophils to prohealing macrophages and proangiogenic nonmyeloid cell populations 3 days after MI (Figure 5, D-F). This reduction in neutrophils and M1-like macrophages may contribute to the reduced tissue remodeling observed following SYN0012 treatment. Indeed, reduced neutrophil influx has been shown to improve cardiac remodeling and prolong survival after MI by reducing proinflammatory signaling and promoting macrophage polarization toward a proreparative M2-like phenotype $(25,26)$. High neutrophil-to-lymphocyte ratios (NLRs) have been shown to be a reliable predictor of myocardial damage following acute coronary events (27). At 3 days after MI, we observed a slight reduction in NLR in the blood with SYN0012 treatment ( $0.342 \pm 0.038$ vs. $0.402 \pm 0.121$ with IgG2a; $P=0.69, t$ test $)$ but a significant decrease in cardiac NLR $(0.457 \pm 0.068$ vs. $1.458 \pm 0.304$ with IgG2a; 
$P=0.017, t$ test), suggesting that $\mathrm{CDH} 11$ blockade alters the distribution of cardiac immune cell populations in a manner that reduces myocardial damage and leads to preserved cardiac function. Despite showing differences in the heart, the percentages of myeloid cells and proangiogenic cells circulating in the peripheral blood were not significantly different 3 days after MI (Supplemental Figure 13). In fact, the ratio of M1/ M2-like macrophages in the blood was significantly increased, suggesting that the SYN0012 likely has an effect on the preferential recruitment of proangiogenic cells and M2-like macrophages to the heart. In light of these findings, future studies could test whether expression of adhesion molecules such as ICAM-1 or selectins expressed by circulating leukocytes and cardiac cells are responsible for an increase in cell recruitment. Alternatively, the unique mechanical environment within the heart may preferentially affect proliferation of recruited immune cells in a CDH11-dependent manner. Indeed, CDH11 deficiency has been shown to reduce macrophage recruitment, migration, proliferation, and expression of M2-macrophage markers — such as ARG1 and CD206 - in pulmonary fibrosis (28).

We observed a reduction in Il6 transcription with SYN0012 treatment at 3 days after infarct, primarily in non-CMs (Figure 6A). IL-6 is a proinflammatory cytokine that has been shown to have a multifaceted role in cardiovascular disease. IL-6 signaling has been shown to promote the infiltration, migration, and polarization of macrophages as well as the activation of myofibroblasts (7, 29-31). Conversely, there is also evidence that blocking IL-6 leads to worsened outcomes after ischemic injury by reducing CM viability and angiogenesis (32-34). By leveraging CDH11 to selectively target non-CMs in the heart, we have identified a therapeutic strategy that effectively limits the negative cell activating effects of IL- 6 without interfering with its function in CMs. The decrease in Il6 transcription, and the corresponding reduction in IL-6 expression in infarcted area of representative hearts, was evident at 3 days after infarct (Figure 6, A and B). This is consistent with the time point of increased inflammatory macrophages and neutrophils in the heart (Figure 1, D-F) and the transition from the inflammatory to proliferative phases of healing. As such, there has been an increasing interest in designing treatment strategies around the role of macrophages - and the associated secreted factors and proinflammatory signaling — in the process of healing and cardiac remodeling after MI (35-38).

We hypothesized that CDH11 regulates the interactions between CFs and macrophages in the heart after MI, noting that macrophage activity has been shown to alter CF function in the ischemic heart independent of CDH11 (39). Interactions between macrophages and CFs have been reported to regulate IL-6 expression in response to TGF- $\beta 1$ signaling and fibrosis (18). CDH11-dependent CF-macrophage interactions in the lungs have also been shown to create a self-sustaining profibrotic niche via local production of latent TGF- $\beta 1$ by macrophages that can be activated by myofibroblasts (20). TGF- $\beta 1$ is a key signal to trigger the resolution of inflammation and initiation of fibrotic remodeling after MI $(40,41)$. We found decreased Il6 transcription and protein expression - a hallmark of CF-macrophage interactions - in SYN0012-treated hearts, relative to IgG2a, at 3 days after MI (Figure 6, A and B). Our coculture data confirm that interactions between macrophages and CFs promote the expression of IL-6 through a partially CDH11-dependent mechanism (Figure 7, A and B). The reduction in the number of macrophages in the heart after 3 days is likely responsible for the reduction in IL-6 expression and the beneficial modulation of both inflammation and fibrosis observed with SYN0012 treatment.

Interestingly, following CDH11 blockade we observed a reduction in transcription of both $\mathrm{Fg} 2$ and Vegfal, two proangiogenic genes often associated with improved revascularization and better outcomes following MI (42-45). However, we also observed an increase in muscularized vessels (i.e., arterioles) in infarcted areas of SYN0012-treated hearts at 21 days after MI; this corresponds to a time when angiogenic gene expression has run its course (46). We speculate that SYN0012 may either preserve native vasculature or hasten maturation of new vasculature, such that the hypoxic conditions that typically drive angiogenic signaling are reduced (47). Blocking CDH11 in endothelial cells could also attenuate endothelial-to-mesenchymal transitions, potentially contributing to preservation of the native vasculature $(10,48)$. The proportion of CECs in the heart over both time points was slightly reduced with SYN0012 treatment, but the increased recruitment of proangiogenic cells, combined with a reduction in early inflammatory cell infiltration, may contribute to a restoration or preservation of infarct vascularization (Figure 5).

Overall, our data reveal that CDH11 engagement plays important roles in the recruitment of specific BMDC populations to the heart after MI that work in concert to promote persistent collagen deposition and fibrotic remodeling by activated myofibroblasts. Importantly, targeting CDH11 with SYN0012 in mice does not prevent the acute inflammatory and reparative response, but rather it selectively acts on several 
cell populations to reduce the duration of inflammation and the extent of fibrotic remodeling resulting in a smaller, more stable infarct. We believe that this effect is mediated, in part, by limiting early recruitment of myeloid lineage BMDCs - including neutrophils and M1-like macrophages - and by reducing macrophage-induced expression of IL- 6 by CFs. Further, SYN0012 treatment promotes recruitment of proangiogenic immune cells to the heart and results in the preservation of arterioles in the infarct area. Taken together, this work has identified $\mathrm{CDH} 11$ as a potential therapeutic target to reduce inflammatory and fibrotic remodeling following MI by selectively targeting a host of active non-CMs responsible for the initiation and progression of cardiac fibrosis and heart failure.

\section{Methods}

Further information and expanded methods can be found in the Supplemental Methods.

Mice. Cdh11-deficieint mice on a mixed C57BL/6 and C129 background were initially obtained from R. Civitelli (Washington University School of Medicine, St. Louis, Missouri, USA), and $C d h 11^{+/+}, C d h 11^{+/-}$, and $C d h 11^{-1-}$ littermate controls were obtained from $C d h 11^{+/-}$breeding pairs. Wild-type C57BL/6J mice (for antibody treatment studies) and B6.SJL-Ptprc $P e p c^{b}$ /BoyJ mice expressing the CD45.1 allele (for bone marrow transplantation studies) were purchased from The Jackson Laboratory. All genotypes were maintained on their respective backgrounds for more than 10 generations.

Animal studies. MI was induced in 12- to 16-week-old male mice by permanent coronary artery ligation, as previously described (24). For antibody treatments, mice were administered either $10 \mathrm{mg} / \mathrm{kg}$ CDH11 functional blocking antibody (SYN0012; with permission from Roche) or an isotype control antibody (IgG2a) resuspended in sterile saline. Antibodies were delivered by i.p. injection every 4 days, beginning 1 day after surgery, with the last treatment given on day 17 after infarct. For bone marrow transplantation studies, 6-week-old B6.SJL-Ptprc ${ }^{a} P e^{b}$ /BoyJ mice expressing the CD45.1 allele were lethally irradiated with a $10-\mathrm{Gy}$ split dose from a $\mathrm{Cs}^{137}$ source. Bone marrow from age- and sex-matched $C d h 11^{+/+}, C d h 11^{+/-}$, and $C d h 11^{-/-}$donors was isolated and transplanted into irradiated recipients by retro-orbital injection $\left(1 \times 10^{6}\right.$ cells in $\left.100 \mu \mathrm{L}\right)$ within 24 hours of irradiation. Transplant efficiency was confirmed by flow cytometry of isolated bone marrow showing simultaneous expression of the donor CD45.2 allele and absence of the original CD45.1 allele (Supplemental Figure 6). To allow for reconstitution of the bone marrow, recipient mice received MI by permanent LAD ligation 6 weeks after transplantation (Figure 2E). Mice were euthanized by $\mathrm{CO}_{2}$ inhalation in accordance with university guidelines at $3,7,21$, and up to 56 days after infarct for further processing.

Echocardiography. EF, LV mass, and LV volume in both systole and diastole were measured from short-axis cardiac M-mode images captured on a Vevo 2100 small-animal ultrasound system (VisualSonics). A minimum of 6 independent measures of LV diameter and wall thickness were used to calculate metrics of cardiac function and geometry for each mouse at each time point. Echocardiographic measurements cardiac structure and function were made just prior to MI (baseline) and at days 7, 21, and 56 days after surgery. Mice with an EF reduced by less than $5 \%$ or greater than $70 \%$ at 7 days after MI were excluded from subsequent analyses.

Cell studies. For separation of CMs from non-CMs, slow perfusion of whole hearts with a $2 \%$ collagenase solution was used to digest the ECM and isolate cells, which were separated into CM and non-CM fractions by centrifugation for 10 minutes at $90 \mathrm{~g}$ (49). CFs were isolated from 8-week-old $\mathrm{Cdh} 11^{+/+}$and Cdh $11^{-1-}$ mice bred onto the Immorto mouse line (to allow for higher passage numbers). Hearts were isolated, minced, and digested in a $2 \%$ collagenase solution supplemented with trypsin for the last 10 minutes of a 40-minute digest. Cells were then rinsed with PBS, transferred to gelatin-coated plates, and cultured in DMEM supplemented with $10 \% \mathrm{FBS}, 1 \%$ penicillin/strep, and IFN- $\gamma$ at $33^{\circ} \mathrm{C}$ to maintain the immortalized phenotype. Prior to experimental use, cells were replated in DMEM supplemented with 10\% FBS and $1 \%$ penicillin/strep and grown at $37^{\circ} \mathrm{C}$ for 48 hours to deactivate the immortalized gene. Macrophage exfiltration was stimulated by i.p. injection of $1 \mathrm{~mL}$ of $4 \%$ thioglycollate media into C57BL/ 6 mice. After 72 hours, mice were sacrificed, and the i.p. cavity was flushed with $10 \mathrm{~mL}$ cold RPMI media to collect the cells. After washing in cold PBS, cells were plated in RPMI media supplemented with $10 \%$ FBS on tissue culture plastic and allowed to adhere for 1 hour. Nonadherent cells were then rinsed away, and all remaining cells were taken to be macrophages (50). For CF-macrophage coculture experiments, 50,000 CFs were plated in each well of a 12-well plate and allowed to adhere for 20 minutes prior to exposure to media containing between 0 and 50,000 macrophages. Cell suspensions were diluted to a final volume of $1.3 \mathrm{~mL}$ 
per well. For antibody treatments, samples were incubated with antibody $(10 \mu \mathrm{g} / \mathrm{ml}$ of either SYN0012 or IgG2a) for 15 minutes before plating. After 48 hours in culture, conditioned media were removed from each well and IL-6 secretion was measured with a DuoSet mouse IL-6 ELISA (R\&D Systems), according to the manufacturer's instructions. After boiling, $100 \mu 1$ of each sample was added in duplicate and compared against a provided standard (Figure $7, \mathrm{~A}$ and $\mathrm{B}$ ).

Flow cytometry. Cells from the hearts and peripheral blood of sham or MI animals were isolated at 3 and 7 days after surgery and immediately placed in a solution of ice-cold FACS buffer (5\% FBS in PBS). Hearts were minced and digested in $1.4 \mathrm{mg} / \mathrm{mL}$ of type II collagenase solution in HBSS for 30 minutes at $37^{\circ} \mathrm{C}$. Isolated cells were filtered through a $70-\mu \mathrm{m}$ cell strainer into room temperature red blood cell lysis buffer prior to staining with DAPI (1:100,000; Thermo Fisher Scientific) to label dead cells and conjugated antibodies for Ter-119 (1:100; violetFluor 450 clone TER-119; Tonbo Biosciences), CD45.2 (1:100; PerCP-Cy5.5 clone 104; Tonbo Biosciences), CD31 (1:100; PE-Cy7 clone MEC13.3; BioLegend), CD11b (1:100; PE clone M1/70; eBioscience), CD206 (1:100; APC clone MR6F3; eBioscience), CD86 (1:100; FITC clone GL1; eBioscience), and Gr-1 (1:100; FITC clone RB6-8C5; eBioscience). Staining for $\mathrm{CDH} 11$ was performed in a 2-step process with an unconjugated primary antibody (1:100; clone 23C6; from M. Brenner [Division of Rheumatology, Immunology, Allergy, Brigham and Women's Hospital and Harvard Medical School, Boston, Massachusetts, USA] [ref. 51]), followed by a PE secondary antibody (1:100; PE clone; RMG1-1; BioLegend). Using this strategy, we identified BMDCs $\left(\mathrm{CD} 45^{+}\right)$, CECs $\left(\mathrm{CD} 45^{-} \mathrm{CD} 11 \mathrm{~b}^{-} \mathrm{CD} 31^{+}\right)$, and $\mathrm{CMCs}\left(\mathrm{CD} 45^{-} \mathrm{CD} 11 \mathrm{~b}^{-} \mathrm{CD} 31^{-}\right)$- primarily myofibroblasts. Within the $\mathrm{BMDC}$ population, we gated for bone marrow-derived proangiogenic cells $\left(\mathrm{CD} 45^{+} \mathrm{CD} 11 \mathrm{~b}-\mathrm{CD} 31^{+}\right)$, myeloid lineage cells $\left(\mathrm{CD} 45^{+} \mathrm{CD} 11 \mathrm{~b}^{+}\right)$, and nonmyeloid lineage cells $\left(\mathrm{CD} 45^{+} \mathrm{CD} 11 \mathrm{~b}^{-} \mathrm{CD} 31^{-}\right)$. Within the myeloid cell population, we identified various subpopulations, including neutrophils $\left(\mathrm{CD} 45^{+} \mathrm{CD}\right.$ $\left.11 b^{+} \mathrm{Gr}-1 / \mathrm{CD} 86^{\text {hi }}\right)$, eosinophils $\left(\mathrm{CD} 45^{+} \mathrm{CD} 11 \mathrm{~b}^{+} \mathrm{Gr}-1 / \mathrm{CD} 86^{\text {lo }} \mathrm{SSC} \mathrm{h}^{\mathrm{hi}}\right)$, monocytes $\left(\mathrm{CD} 45^{+} \mathrm{CD} 11 \mathrm{~b}^{+} \mathrm{Gr}-1 /\right.$ $\left.\mathrm{CD} 6^{\text {lo }} \mathrm{SSC}^{\mathrm{lo}}\right)$, and macrophages $\left(\mathrm{CD} 45^{+} \mathrm{CD} 11 \mathrm{~b}^{+} \mathrm{Gr}-1 / \mathrm{CD}^{2} 6^{\mathrm{int}}\right)(17)$. We further assessed macrophage polarization by gating for proinflammatory (M1-like) macrophages (CD45 $\left.{ }^{+} \mathrm{CD} 11 \mathrm{~b}^{+} \mathrm{Gr}-1 / \mathrm{CD}^{2} 6^{\text {int }} \mathrm{CD} 206^{-}\right)$ and proresolving (M2-like) macrophages $\left(\mathrm{CD} 45^{+} \mathrm{CD} 11 \mathrm{~b}^{+} \mathrm{Gr}-1 / \mathrm{CD} 6^{\text {int }} \mathrm{CD} 206^{+}\right)$(Supplemental Figure 1). The NLR was computed by dividing the number of positively identified neutrophils by the number of nonmyeloid BMDCs in each sample (27). Note that antibodies specific for CD45.1 (1:100; PE clone A20; BD Biosciences) and CD45.2 (1:100; FITC clone 104; BD Biosciences) were used for assessment of bone marrow engraftment efficiency (Supplemental Figure 6). More details about the gating strategy are presented in the Supplemental Methods (Supplemental Figures 1 and 2).

Histology. Following euthanasia, a subset of hearts was dissected into PBS, weighed (Supplemental Figure 10, $\mathrm{A}$ and $\mathrm{B}$ ), and submerged briefly in a $\mathrm{KCl}$ solution to relax the CMs. Relaxed hearts were then bisected along the transverse plane (orthogonal to the long axis of the heart), embedded in OCT media, and frozen. Frozen blocks were cryosectioned into $10-\mu \mathrm{m}$ sections, mounted onto glass slides, and stored at $-20^{\circ} \mathrm{C}$. Prior to staining with Masson's trichrome (MilliporeSigma) to identify regions of healthy myocardium (red/ pink), collagen/ECM deposition (blue), and cell nuclei (black), slides were brought to room temperature, OCT media were dissolved in PBS, and sections were fixed in Bouin's solution. To quantify infarct morphology from Masson's trichrome-stained sections, a semiautomated image-processing pipeline was developed based on local ventricular thickness measurements and color segmentation (Supplemental Figure 9 and refs. 52, 53). Image quantification was performed on 3 sections per heart separated by at least $300 \mu \mathrm{m}$.

Immunohistochemistry. Frozen slides were brought to room temperature, OCT media were dissolved in $\mathrm{PBS}$, and tissue sections were fixed in $4 \%$ paraformaldehyde with $0.3 \%$ Triton- $\mathrm{X}$ for 10 minutes followed by blocking in 1\% BSA in PBS for 1 hour. Sections were then stained for $\alpha \mathrm{SMA}$ (Cy3, c1A4; MilliporeSigma), CD31 (Alexa Fluor 594, c390; Biolegend), CD45 (FITC CD45.2; BD Pharmingen), or IL-6 (polyclonal ab6672; Abcam). Sections stained with nonconjugated antibodies were incubated at a 1:100 dilution in $1 \%$ BSA overnight at $4^{\circ} \mathrm{C}$. Sections were then rinsed with PBS and incubated with fluorescently tagged secondary antibody (goat anti-rabbit Alexa Fluor 647, A27040; Thermo Fisher Scientific) for 1 hour at a 1:300 dilution in $1 \%$ BSA. Sections stained with directly conjugated antibodies were incubated at a 1:100 dilution in $1 \%$ BSA for 1 hour at room temperature. Stained slides were mounted in ProLong Gold with DAPI to visualize cell nuclei. Muscularized vessels smaller than approximately $100 \mu \mathrm{m}$ in diameter and myofibroblasts (defined as $\alpha \mathrm{SMA}^{+}$cells not colocalized with endothelial cells) were manually counted, and IL-6 staining in the infarcted region was quantified by measuring the average fluorescent intensity within the infarct area (manually segmented in ImageJ [NIH]) and the myocardium as a whole (Supplemental Figure 15). 
AFM. Infarct stiffness was quantified by AFM using the Biocatalyst AFM and the peak force quantitative nanomechanical mapping mode developed by Bruker. Tissue sections were brought to room temperature, and OCT media were dissolved in PBS and blocked in 10\% FBS for 20 minutes prior to scanning with the AFM. All measurements were made in PBS and were acquired from at least 5 separate $10 \times 10 \mu \mathrm{m}^{2}$ areas from a minimum of 2 different sections per mouse (Supplemental Figure 8).

Collagen gel contraction. CFs were diluted in a $1.28 \mathrm{mg} / \mathrm{mL}$ collagen solution derived from PureCol (Advanced Biomatrix) to a final concentration of 250,000 cells $/ \mathrm{mL}$ and were poured into a Teflon ring in a suspension well. After polymerizing for 1 hour, DMEM supplemented with 10\% FBS and 1\% penicillin/strep was added to release the collagen gel. Images were acquired immediately after release and at multiple times over the next 48 hours. Gel contraction was assessed by measuring surface area using ImageJ. For comparison of IgG2a and SYN0012 treatments, antibody was added to the cell/gel mixture at a final concentration of 20 $\mu \mathrm{g} / \mathrm{ml}$ prior to pouring; media added to the well also contained $10 \mu \mathrm{g} / \mathrm{ml}$ antibody (Supplemental Figure 11).

$q P C R$. Hearts from sham and MI animals were isolated under RNAse-free conditions and immediately flash frozen. For isolation of mRNA and transcriptional analysis of specific profibrotic and inflammatory genes of interest, samples were subsequently thawed and lysed in TRIZOL with chloroform-induced phase separation according to the manufacturer's instructions. Similarly, cells from CF-macrophage cocultures were lysed in TRIZOL for isolation of mRNA (Figure 7, C-E, and Supplemental Figure 16). cDNA was synthesized using the Superscript IV kit (Invitrogen) using 500 ng mRNA. Real-time qPCR was used to amplify targets from the cDNA using SYBR green master mix (Bio-Rad) and specific primer sets of interest (Supplemental Table 1). The BIO-RAD CFX96 C1000 system was used to quantify gene transcription in each sample, relative to Gapdh (forward: ATGACAATGAATACGGCTACAG and reverse: TCTCTTGCTCAGTGTCCTTG). For all in vivo transcription levels, after MI samples were normalized to the average of all 3 and 7 day sham values (Supplemental Figure 14).

Statistics. Data throughout the manuscript are presented as mean \pm SEM. Two-tailed Student's $t$ tests were used to determine significant effects between 2 groups. One- or two-way ANOVAs and linear mixed-effects models with restricted maximum likelihood were used to determine statistical differences between different sample groups over multiple time points, with post hoc Holms-Sidak's tests used to account for multiple comparisons. An overall value of $P<0.05$ was considered significant in all statistical comparisons. Specific details regarding statistical tests performed for each measured variable are outlined in the Supplemental Methods.

Study approval. All animal procedures were approved by the Institutional Animal Care and Use Committee at Vanderbilt University.

\section{Author contributions}

AKS designed experiments, performed antibody treatments, and processed qPCR, AFM, histology, immunostaining, and coculture data. MRB designed experiments, performed the flow cytometry experiments, refined the flow cytometry gating strategy, and developed the automated histological image processing codes. CRC designed experiments, collected echocardiography data from Cdh11-deficient animals, performed bone marrow transplantations, and performed flow cytometry experiments with antibody treatment. QZ performed MI surgeries. LHS, AKH, TLF, and HL participated in discussions regarding planning of experiments and provided expertise. SMM helped design the flow cytometry experiments and analysis. WDM designed experiments and supervised the study. AKS, MRB, and WDM wrote and revised the manuscript. All authors were involved in the analysis and interpretation of data and have read and approved the manuscript.

\section{Acknowledgments}

We thank the Vanderbilt Cardiovascular Physiology Core for performing echocardiographic assessments for this study. We thank Claire Lafferty and Joshua Bender for assisting in the collection of the AFM and histological data. We also thank Roche (Dominik Hartl and Uwe Junker, Roche Pharma Research and Early Development, Immunology, Inflammation and Infectious Diseases Discovery and Translational Area and the Cadherin-11 team, Roche Innovation Center Basel, Switzerland) for supplying the blocking antibody, SYN0012. This work was funded by the National Institutes of Health (HL135790, HL115103, HL146951, and HL007411), the National Science Foundation (1055384 and DGE-0909667), the American Heart Association (15PRE25710333), and the Leducq Foundation. 
Address correspondence to: W. David Merryman, Room 9445D MRB4, 2213 Garland Avenue, Nashville, Tennessee 37212, USA. Phone: 615.322.7219; Email: david.merryman@vanderbilt.edu.

1. Benjamin EJ, et al. Heart Disease and Stroke Statistics-2018 Update: A Report From the American Heart Association. Circulation. 2018;137(12):e67-e492.

2. Dick SA, et al. Self-renewing resident cardiac macrophages limit adverse remodeling following myocardial infarction. Nat Immunol. 2019;20(1):29-39.

3. Boudoulas KD, Hatzopoulos AK. Cardiac repair and regeneration: the Rubik's cube of cell therapy for heart disease. Dis Model Mech. 2009;2(7-8):344-358.

4. Richardson WJ, Holmes JW. Why Is Infarct Expansion Such an Elusive Therapeutic Target? J Cardiovasc Transl Res. 2015;8(7):421-430.

5. Gombozhapova A, et al. Macrophage activation and polarization in post-infarction cardiac remodeling. J Biomed Sci. 2017;24(1):13

6. Ikeuchi M, et al. Inhibition of TGF-beta signaling exacerbates early cardiac dysfunction but prevents late remodeling after infarction. Cardiovasc Res. 2004;64(3):526-535.

7. Fuchs M, et al. Role of interleukin-6 for LV remodeling and survival after experimental myocardial infarction. FASEB J. 2003;17(14):2118-2120.

8. Frangogiannis NG. The inflammatory response in myocardial injury, repair, and remodelling. Nat Rev Cardiol. 2014;11(5):255-265.

9. Chang SK, et al. Cadherin-11 regulates fibroblast inflammation. Proc Natl Acad Sci USA. 2011;108(20):8402-8407.

10. Schneider DJ, et al. Cadherin-11 contributes to pulmonary fibrosis: potential role in TGF- $\beta$ production and epithelial to mesenchymal transition. FASEB J. 2012;26(2):503-512.

11. Clark CR, Bowler MA, Snider JC, Merryman WD. Targeting Cadherin-11 Prevents Notch1-Mediated Calcific Aortic Valve Disease. Circulation. 2017;135(24):2448-2450.

12. Lee YC, et al. Inhibition of cell adhesion by a cadherin-11 antibody thwarts bone metastasis. Mol Cancer Res. 2013;11(11):1401-1411.

13. ${ }^{\circ}$. Cadherin-11 expression is upregulated in invasive human breast cancer. Oncol Lett. 2016;12(6):4393-4398.

14. Thompson SA, et al. Acute slowing of cardiac conduction in response to myofibroblast coupling to cardiomyocytes through N-cadherin. J Mol Cell Cardiol. 2014;68:29-37.

15. Assefnia S, et al. Cadherin-11 in poor prognosis malignancies and rheumatoid arthritis: common target, common therapies. Oncotarget. 2014;5(6):1458-1474.

16. Hutcheson JD, et al. Cadherin-11 regulates cell-cell tension necessary for calcific nodule formation by valvular myofibroblasts. Arterioscler Thromb Vasc Biol. 2013;33(1):114-120.

17. Rose S, Misharin A, Perlman H. A novel Ly6C/Ly6G-based strategy to analyze the mouse splenic myeloid compartment. Cytometry A. 2012;81(4):343-350.

18. Ma F, et al. Macrophage-stimulated cardiac fibroblast production of IL-6 is essential for TGF $\beta /$ Smad activation and cardiac fibrosis induced by angiotensin II. PLoS ONE. 2012;7(5):e35144.

19. Bowler MA, Bersi MR, Ryzhova LM, Jerrell RJ, Parekh A, Merryman WD. Cadherin-11 as a regulator of valve myofibroblast mechanobiology. Am J Physiol Heart Circ Physiol. 2018;315(6):H1614-H1626.

20. Lodyga M, et al. Cadherin-11-mediated adhesion of macrophages to myofibroblasts establishes a profibrotic niche of active TGF-ß. Sci Signal. 2019;12(564):eaao3469.

21. Protti A, et al. Bone marrow transplantation modulates tissue macrophage phenotype and enhances cardiac recovery after subsequent acute myocardial infarction. J Mol Cell Cardiol. 2016;90:120-128.

22. Horikawa K, Radice G, Takeichi M, Chisaka O. Adhesive subdivisions intrinsic to the epithelial somites. Dev Biol. 1999;215(2):182-189.

23. Row S, Liu Y, Alimperti S, Agarwal SK, Andreadis ST. Cadherin-11 is a novel regulator of extracellular matrix synthesis and tissue mechanics. J Cell Sci. 2016;129(15):2950-2961.

24. Gao E, et al. A novel and efficient model of coronary artery ligation and myocardial infarction in the mouse. Circ Res. 2010;107(12):1445-1453.

25. Kubota A, Suto A, Suzuki K, Kobayashi Y, Nakajima H. Matrix metalloproteinase-12 produced by Ly6C $C^{\text {low }}$ macrophages prolongs the survival after myocardial infarction by preventing neutrophil influx. J Mol Cell Cardiol. 2019;131:41-52.

26. Horckmans M, et al. Neutrophils orchestrate post-myocardial infarction healing by polarizing macrophages towards a reparative phenotype. Eur Heart J. 2017;38(3):187-197.

27. Chen C, et al. Neutrophil to lymphocyte ratio as a predictor of myocardial damage and cardiac dysfunction in acute coronary syndrome patients. Integr Med Res. 2018;7(2):192-199.

28. To S, Agarwal SK. Macrophages and the regulation of pulmonary fibrosis by Cadherin-11. J Immunol. 2018;200(Suppl 1):49.14.

29. Zhang C, Li Y, Wu Y, Wang L, Wang X, Du J. Interleukin-6/signal transducer and activator of transcription 3 (STAT3) pathway is essential for macrophage infiltration and myoblast proliferation during muscle regeneration. $J$ Biol Chem. 2013;288(3):1489-1499.

30. Fernando MR, Reyes JL, Iannuzzi J, Leung G, McKay DM. The pro-inflammatory cytokine, interleukin-6, enhances the polarization of alternatively activated macrophages. PLOS ONE. 2014;9(4):e94188.

31. Meléndez GC, McLarty JL, Levick SP, Du Y, Janicki JS, Brower GL. Interleukin 6 mediates myocardial fibrosis, concentric hypertrophy, and diastolic dysfunction in rats. Hypertension. 2010;56(2):225-231.

32. Müller J, et al. Interleukin-6-dependent phenotypic modulation of cardiac fibroblasts after acute myocardial infarction. Basic Res Cardiol. 2014;109(6):440.

33. Huang M, Yang D, Xiang M, Wang J. Role of interleukin-6 in regulation of immune responses to remodeling after myocardial infarction. Heart Fail Rev. 2015;20(1):25-38.

34. Mayfield AE, et al. Interleukin-6 Mediates Post-Infarct Repair by Cardiac Explant-Derived Stem Cells. Theranostics. 2017;7(19):4850-4861. 
35. Chen B, Frangogiannis NG. Macrophages in the Remodeling Failing Heart. Circ Res. 2016;119(7):776-778.

36. Frangogiannis NG. Emerging roles for macrophages in cardiac injury: cytoprotection, repair, and regeneration. J Clin Invest. 2015;125(8):2927-2930.

37. Ma Y, Mouton AJ, Lindsey ML. Cardiac macrophage biology in the steady-state heart, the aging heart, and following myocardial infarction. Transl Res. 2018;191:15-28.

38. Bajpai G, et al. The human heart contains distinct macrophage subsets with divergent origins and functions. Nat Med. 2018;24(8):1234-1245.

39. Shiraishi M, et al. Alternatively activated macrophages determine repair of the infarcted adult murine heart. J Clin Invest. 2016;126(6):2151-2166.

40. Nah DY, Rhee MY. The inflammatory response and cardiac repair after myocardial infarction. Korean Circ J. 2009;39(10):393-398.

41. Frantz S, et al. Transforming growth factor beta inhibition increases mortality and left ventricular dilatation after myocardial infarction. Basic Res Cardiol. 2008;103(5):485-492.

42. Paik DT, et al. Wnt10b Gain-of-Function Improves Cardiac Repair by Arteriole Formation and Attenuation of Fibrosis. Circ Res. 2015;117(9):804-816.

43. Virag JA, Rolle ML, Reece J, Hardouin S, Feigl EO, Murry CE. Fibroblast growth factor-2 regulates myocardial infarct repair: effects on cell proliferation, scar contraction, and ventricular function. Am J Pathol. 2007;171(5):1431-1440.

44. Yang Y, et al. Modified VEGF targets the ischemic myocardium and promotes functional recovery after myocardial infarction. $J$ Control Release. 2015;213:27-35.

45. Carlsson L, et al. Biocompatible, Purified VEGF-A mRNA Improves Cardiac Function after Intracardiac Injection 1 Week Post-myocardial Infarction in Swine. Mol Ther Methods Clin Dev. 2018;9:330-346.

46. Zhao T, Zhao W, Chen Y, Ahokas RA, Sun Y. Vascular endothelial growth factor (VEGF)-A: role on cardiac angiogenesis following myocardial infarction. Microvasc Res. 2010;80(2):188-194.

47. Orlandini M, Oliviero S. In fibroblasts Vegf-D expression is induced by cell-cell contact mediated by cadherin-11. J Biol Chem. 2001;276(9):6576-6581.

48. Aisagbonhi O, Rai M, Ryzhov S, Atria N, Feoktistov I, Hatzopoulos AK. Experimental myocardial infarction triggers canonical Wnt signaling and endothelial-to-mesenchymal transition. Dis Model Mech. 2011;4(4):469-483.

49. O'Connell TD, Rodrigo MC, Simpson PC. Isolation and Culture of Adult Mouse Cardiac Myocytes. In: Vivanco F, ed. Cardiovascular Proteomics. Clifton, New Jersey: Humana Press; 2007: 271-96.

50. Zhang X, Goncalves R, Mosser DM. The isolation and characterization of murine macrophages. Curr Protoc Immunol. 2008; Chapter 14:Unit 14.1.

51. Chang SK, et al. Stromal cell cadherin-11 regulates adipose tissue inflammation and diabetes. J Clin Invest. 2017;127(9):3300-3312

52. Yezzi AJ, Prince JL. An Eulerian PDE approach for computing tissue thickness. IEEE Trans Med Imaging. 2003;22(10):1332-1339.

53. Bersi MR, Khosravi R, Wujciak AJ, Harrison DG, Humphrey JD. Differential cell-matrix mechanoadaptations and inflammation drive regional propensities to aortic fibrosis, aneurysm or dissection in hypertension. J $R$ Soc Interface. 2017;14(136):20170327 